\title{
A statistical mechanics approach to mixing in stratified fluids
}

\author{
A. Venaille ${ }^{1, \dagger}$, L. Gostiaux ${ }^{2}$ and J. Sommeria ${ }^{3}$ \\ ${ }^{1}$ Univ Lyon, Ens de Lyon, Univ Claude Bernard, CNRS, Laboratoire de Physique, F-69342 Lyon, France \\ ${ }^{2}$ LMFA UMR 5509 CNRS, Université de Lyon, Ecole Centrale 69130 Écully Lyon, France \\ ${ }^{3}$ LEGI, CNRS, Université de Grenoble, CS 4070038058 Grenoble Cedex 9, France
}

(Received 6 March 2016; revised 25 October 2016; accepted 28 October 2016; first published online 1 December 2016)

Predicting how much mixing occurs when a given amount of energy is injected into a Boussinesq fluid is a long-standing problem in stratified turbulence. The huge number of degrees of freedom involved in these processes renders extremely difficult a deterministic approach to the problem. Here we present a statistical mechanics approach yielding a prediction for a cumulative, global mixing efficiency as a function of a global Richardson number and the background buoyancy profile. Assuming random evolution through turbulent stirring, the theory predicts that the inviscid, adiabatic dynamics is attracted irreversibly towards an equilibrium state characterised by a smooth, stable buoyancy profile at a coarse-grained level, upon which are fine-scale fluctuations of velocity and buoyancy. The convergence towards a coarse-grained buoyancy profile different from the initial one corresponds to an irreversible increase of potential energy, and the efficiency of mixing is quantified as the ratio of this potential energy increase to the total energy injected into the system. The remaining part of the energy is lost into small-scale fluctuations. We show that for sufficiently large Richardson number, there is equipartition between potential and kinetic energy, provided that the background buoyancy profile is strictly monotonic. This yields a mixing efficiency of 0.25 , which provides statistical mechanics support for previous predictions based on phenomenological kinematics arguments. In the general case, the cumulative, global mixing efficiency predicted by the equilibrium theory can be computed using an algorithm based on a maximum entropy production principle. It is shown in particular that the variation of mixing efficiency with the Richardson number strongly depends on the background buoyancy profile. This approach could be useful to the understanding of mixing in stratified turbulence in the limit of large Reynolds and Péclet numbers.

Key words: stratified flows, turbulence modelling, turbulent mixing

\section{Introduction}

The large-scale stratification and dynamics of the oceans depend crucially on localised turbulent mixing events (Wunsch \& Ferrari 2004; Thorpe 2005). These mixing processes occur on temporal and spatial scales much smaller than the current

$\dagger$ Email address for correspondence: antoine.venaille@ens-lyon.fr 
resolutions of general circulation models and must therefore be parameterised (Large, McWilliams \& Doney 1994). It is essential for that purpose to know how much mixing occurs when stratification is stirred by a turbulent flow (Hopfinger 1987; Fernando 1991; Staquet \& Sommeria 2002; Peltier \& Caulfield 2003; Ivey, Winters $\&$ Koseff 2008). More precisely, which fraction of the injected energy is lost through a direct turbulent kinetic energy cascade and viscous dissipation, which fraction contributes to modifying the background stratification and what is the resulting vertical buoyancy profile? Here we propose to use statistical mechanics as a guideline for the understanding of turbulent stirring and mixing in a stratified fluid.

Equilibrium statistical mechanics counts the available states of the system with given constraints based on conservation laws. Under random evolution, the system is expected to reach the macroscopic state, which corresponds to the maximum number of microscopic configurations. In this paper, the macroscopic quantity to be determined by the theory is the partition between kinetic and potential energy, as well as the corresponding mean (coarse-grained) vertical buoyancy profile. The microscopic configurations will consists of the set of all possible buoyancy fields and non-divergent velocity fields, and the constraints will be provided by dynamical invariants of the inviscid and adiabatic fluid.

The application of equilibrium statistical mechanics theory to systems described by continuous fields is however problematic; see e.g. Pomeau (1994). Indeed, such systems are characterised by an infinite number of degrees of freedom, which can lead to an accumulation of energy at small scales, whose divergence can only be avoided by an artificial truncation in Fourier space. Kraichnan (1967) has however explained the energy cascade toward small scales as a trend of the system to approach such an equilibrium. By contrast, in two-dimensional turbulence, statistical equilibrium rather accumulates energy at large scales, which Kraichnan has related to the occurrence of an inverse energy cascade. The statistical equilibrium therefore reveals the trend of the evolution for the actual irreversible turbulent system in the limit of small viscosity. We here follow a similar idea to study mixing in stratified fluids, using however a significantly different statistical mechanics approach.

Instead of considering Galerkin-truncated flows, Onsager (1949) modelled the fluid continuum by a very large but finite set of singular point vortices to explain the self-organisation of two-dimensional turbulent flows as a tendency to reach an equilibrium state, see also Eyink \& Sreenivasan (2006). Extensions of those ideas to the continuous two-dimensional Euler and quasi-geostrophic dynamics have been developed independently by Miller (1990) and Robert \& Sommeria (1991) (MRS hereafter). A similar theory had been previously applied to the Vlasov equations by Lynden-Bell (1967) in order to predict self-organisation in plasma and self-gravitating systems, see e.g. Chavanis (2002). Subsequent work on the theoretical foundations of the approach, as well as on the analytical and numerical computation of equilibrium states, is reviewed in Sommeria (2001), Majda \& Wang (2006), Bouchet \& Venaille (2012). The theory introduces a truncation for the vorticity field, leading to unrealistic vorticity fluctuations at small scales, but it provides quantitative predictions for the mean velocity field at large scales. In the geophysical context, the theory has been used to explain some features of the structure of the Great Red Spot of Jupiter (Turkington et al. 2001; Bouchet \& Sommeria 2002), oceanic rings and jets (Weichman 2006; Venaille \& Bouchet 2011), bottom-trapped oceanic recirculations (Venaille 2012), the stratospheric polar vortex (Prieto \& Schubert 2001), the vertical structure of geostrophic turbulence in stratified quasi-geostrophic turbulence (Merryfield 1998; Schecter 2003; Venaille, Vallis \& Griffies 2012) and the 
structure of the thermocline in the global oceanic circulation (Salmon 2012). One should however keep in mind that statistical equilibrium theory strictly applies to freely evolving flows, while most geophysical situations involve forcing and friction. The equilibrium theory can be relevant to describe the large-scale flow when forcing and dissipation are sufficiently weak (Majda \& Wang 2006; Bouchet \& Simonnet 2009), but the values of conserved quantities are then set by the global balance between forcing and dissipation rather than from initial conditions.

The equilibrium theory has already been derived for several flow systems that permit the existence of a direct energy cascade, such as three-dimensional axisymmetric Euler flows (Naso et al. 2010; Thalabard, Dubrulle \& Bouchet 2014; Thalabard et al. 2015). The theory yields in this case predictions for the energy partition between toroidal and poloidal modes (Thalabard et al. 2014). Similarly, equilibrium theory has been used to predict the energy partition between inertia-gravity waves and vortical modes in shallow water models (Warn 1986; Weichman \& Petrich 2001; Renaud, Venaille \& Bouchet 2016). Here we apply a similar approach to a non-rotating, density-stratified Boussinesq fluid in order to predict the partition between kinetic and potential energy for a given amount of energy injected into the system. Tabak \& Tal (2004) computed the most probable buoyancy field of a two-layer fluid with a prescribed total energy, assuming that the kinetic energy is constant at each height. Our contributions are twofold. First, we generalise their result to arbitrary buoyancy profiles, and obtain the kinetic energy profile as the output of the statistical theory. Second, we use these results to obtain predictions for mixing efficiency in decaying configurations.

How to infer the efficiency of mixing in forced-dissipative or decaying experiments has been carefully addressed in previous studies; see e.g. Winters et al. (1995), Peltier \& Caulfield (2003), Davis Wykes, Hughes \& Dalziel (2015), Salehipour \& Peltier (2015) and references therein. The traditional approach involves direct analyses of the diffusive destruction of small-scale density variance as the experiment proceeds, which in turn requires a separation of the influence of stirring from that of irreversible mixing through application of the Lorenz concept of available potential energy that can be converted into kinetic energy and a base-state potential energy which cannot. It has been demonstrated that the diffusive destruction of small-scale density variance may be represented by the time derivative $\mathcal{M}$ of base-state potential energy plus a small correction due to the action of molecular diffusion on the initial density stratification, a correction that becomes negligible in the limit of high Reynolds number (Winters et al. 1995). The time-dependent efficiency of turbulent mixing may be then computed from the direct numerical simulations as $\eta_{t}=\mathcal{M} /(\mathcal{M}+\epsilon)$ where $\epsilon$ is the rate of viscous kinetic energy dissipation in the fluid domain (Peltier \& Caulfield 2003; Salehipour \& Peltier 2015). This definition of mixing efficiency is global in space since the computation of the base-state potential energy requires a rearrangement of the fluid particle at the domain scale. Using a number of additional assumptions, it may be related to a local mixing efficiency that is often used in oceanography to model an effective diffusivity for diapycnal mixing (Osborn 1980; Hopfinger 1987; Tailleux 2009). In decaying experiments, it is also convenient to define a cumulative mixing efficiency $\eta_{t o t}=\int_{0}^{+\infty} \mathrm{d} t \mathcal{M} / \int_{0}^{+\infty} \mathrm{d} t(\mathcal{M}+\epsilon)$, which measures how much of the total injected energy has been used to irreversibly raise the potential energy of the system in the experiment. In practice, this quantity can easily be inferred in laboratory experiments by measuring the buoyancy profile once all dissipative effects have died out, assuming the initial background stratification and the initial injected energy are known. 
Although the traditional approach to mixing efficiency in stratified turbulence emphasises the role of molecular diffusivity, we argue in this paper that irreversible mixing in decaying configurations can also be addressed within the framework of an inviscid, adiabatic Boussinesq flow model. Indeed, we will see that even if the background buoyancy field remains constant in time, the system is irreversibly attracted towards a state characterised by small-scale buoyancy fluctuations and a concomitant irreversible increase of available potential energy, assuming ergodicity. This irreversibility is due to the fact that an overwhelming number of microscopic configurations are close the most probable state, according to the equilibrium theory. The available potential energy of the equilibrium state could in principle be transferred back into kinetic energy, but this would correspond to the highly improbable escape from the equilibrium state. More precisely, we will show that the probability to observe a state different than the equilibrium state is vanishingly small (it tends to zero as the number of fluid particles tends to infinity). In other words, the so-called available potential energy of the system is statistically not available when the equilibrium state is reached, and we argue that a statistical mixing efficiency can be defined without reference to the molecular diffusion of buoyancy levels. Our working hypothesis is that this statistical mixing efficiency is equivalent to the traditional definition of mixing efficiency in the limit of weak molecular diffusivity.

Applying the statistical mechanics programs to Boussinesq dynamics is done in three steps. The first step is to find relevant phase-space variables. These variables must satisfy a Liouville theorem, and we show in this paper (appendix A) that this is the case of the velocity and buoyancy fields. This ensures that the dynamics is non-divergent in phase space, so that the probability densities expressed in these variables remain constant during the time evolution of the system. The fundamental postulate of equal probability for each microscopic configuration is then consistent with the dynamical evolution. Second, we need to introduce a discretisation of the continuous fields describing the system. This technical step is classical when computing equilibrium states of systems described by deterministic partial differential equations. Once the discrete approximation of the fields is introduced, one can count the microscopic configurations, and the computation of the equilibrium states is rigorous. The third step is to introduce a macroscopic description of the system, and to find the most probable macrostates among all those that satisfy a set of constraints provided by dynamical invariants. Using the equilibrium theory to describe the long-time behaviour of the system finally requires the assumption of ergodicity, i.e. that the system evenly explores phase space. Even if the ergodic assumption may not be fulfilled in actual turbulent flow, computing the equilibria is at least a useful and necessary first step before addressing the out of equilibrium behaviour of the system in more comprehensive studies.

Denoting $H$ the height of the flow domain, $\Delta b$ the typical variations of the background buoyancy profile, $\left(U, L_{t}\right)$ the typical velocity and length scale of turbulence and $(\nu, \kappa)$ the molecular viscosity and diffusivity, the efficiency of mixing depends a priori on four non-dimensional parameters in laboratory or numerical experiments on stratified turbulence: a global Richardson number based on the domain scale $R i=H \Delta b / U^{2}$, the Reynolds number $R e=U L_{t} / \nu$, the Péclet number $P e=U L_{t} / \kappa$ and the ratio $L_{t} / H$ which depends on the energy injection mechanism.

The equilibrium statistical mechanics theory applies to the freely evolving inviscid adiabatic dynamics. Considering such an approach to describe actual stratified turbulence amounts to assuming that the Reynolds number $R e$ and the Péclet number $\mathrm{Pe}$ are sufficiently large, and that the typical time scale to approach the equilibrium 
state is smaller than the typical time scale for the dissipation of energy and buoyancy fluctuations. Independently from statistical mechanics arguments, neglecting molecular effects is a natural assumption in the large Reynolds number limit, which has been proven useful in previous studies on three-dimensional turbulence (Eyink \& Sreenivasan 2006), in which case the observed dissipation rate of energy $\epsilon$ becomes independent from viscosity; see e.g. Vassilicos (2015) and references therein.

Similarly, the independence of the dissipation rate of scalar fluctuations on the molecular diffusivity is a standard hypothesis in turbulence theory, coming back to the generalisation of Kolmogorov arguments by Obukhov (1949) and Corrsin (1951). In the case of a passive scalar, this hypothesis has been supported by experiments (Sreenivasan 1996; Warhaft 2000) and theoretical results (Shraiman \& Siggia 2000; Falkovich, Gawędzki \& Vergassola 2001).

Within the framework of the equilibrium theory, we assume conservation of the total energy and of the global distribution of buoyancy, but we show that part of the energy and that part of the buoyancy fluctuations are irreversibly transferred to small scales once the equilibrium state is reached. Since the amount of kinetic energy and buoyancy fluctuations that are irreversibly transferred to small scales can be computed explicitly within the equilibrium statistical mechanics framework, we argue that the theory makes possible a prediction for the cumulative mixing efficiency, even in the absence of viscosity or molecular dissipation in the model. Our working hypothesis is that those small-scale fluctuations will be smoothed out by molecular effects over a time scale much larger than the relaxation time towards equilibrium.

The paper is organised as follows. The equilibrium statistical mechanics theory is introduced and discussed in the second section. The actual computation of the equilibrium states is discussed in a third section. Application of the theory to predict mixing efficiency in freely evolving flow (decaying turbulence) is discussed in a fourth section. We conclude and summarise the main results in the fifth section. Technical results on the Liouville theorem, on the computation of the macrostate entropy and on the numerical algorithm used to compute the equilibria are presented in two appendices.

\section{Equilibrium statistical mechanics of non-rotating, density-stratified Boussinesq fluids}

\subsection{Dynamical system and invariants}

We consider an inviscid Boussinesq fluid that evolves in a three-dimensional domain $\mathcal{V}_{x}$ of volume $V$, see e.g. Vallis (2006). Spatial coordinates are denoted $\boldsymbol{x}=(x, y, z)$, with $\boldsymbol{e}_{z}$ the vertical unit vector pointing in the upward direction. At each time $t$ the system is described by the buoyancy field $b=g\left(\varrho_{0}-\varrho\right) / \varrho_{0}$, where $\varrho(x, y, z, t)$ is the fluid density, $g$ gravity and $\varrho_{0}$ a reference density, and by the velocity field $\boldsymbol{u}=(u, v, w)$, which is non-divergent:

$$
\nabla \cdot u=0 .
$$

In the absence of diffusivity, the buoyancy field is purely advected by the velocity field

$$
\partial_{t} b+\boldsymbol{u} \cdot \nabla b=0,
$$

and the dynamics of the velocity field is coupled to the buoyancy field through the momentum equation

$$
\partial_{t} \boldsymbol{u}+\boldsymbol{u} \cdot \nabla \boldsymbol{u}=-\frac{1}{\varrho_{0}} \nabla P+b \boldsymbol{e}_{z} .
$$


Equation (2.2) describes the Lagrangian conservation of the buoyancy. It implies the conservation of the global distribution (i.e. histogram) of buoyancy levels

$$
G(\sigma)=\frac{1}{V} \int_{\mathcal{V}_{x}} \mathrm{~d} \boldsymbol{x} \delta(b-\sigma)
$$

expressed as $\mathrm{d} G / \mathrm{d} t=0$. The conservation of $G(\sigma)$ is equivalent to the conservation of all the Casimir functionals $F[b]=\int \mathrm{d} \boldsymbol{x} f(b)$, with $f$ any arbitrary function; see e.g. Potters, Vaillant \& Bouchet (2013). This conservation law is also equivalent to the conservation of the background (or sorted) buoyancy profile $b_{s}(s)$ defined as the buoyancy profile with minimal potential energy using

$$
G\left(b_{s}\right) \mathrm{d} b_{s}=\frac{1}{2 H} \mathrm{~d} z .
$$

Similarly, using (2.1), (2.2) and (2.3) one can show that the total energy of the flow

$$
E=\int_{\mathcal{V}_{x}} \mathrm{~d} \boldsymbol{x}\left(\frac{1}{2} \boldsymbol{u}^{2}-b z\right)+\int_{\mathcal{V}_{x}} \mathrm{~d} \boldsymbol{x} z b_{s}
$$

is another dynamical invariant: $\mathrm{d} E / \mathrm{d} t=0$. Note that the total energy is defined up to a constant, but we have chosen this constant such that the energy vanishes when there is no motion and when the buoyancy field is sorted $\left(E=0\right.$ when $\boldsymbol{u}=0$ and $b=b_{s}$ ).

The Boussinesq equations are characterised by additional dynamical invariants related to the conservation of Ertel potential vorticity, see e.g. Salmon (1998). These invariants are essential to explain the occurrence of inverse cascade and self-organisation of the velocity field occurring in the presence of sufficiently large rotation. However various theoretical and numerical studies indicate that stratified turbulence in the absence of rotation is not influenced significantly by these invariants (Bartello 1995; Waite \& Bartello 2004; Lindborg 2005, 2006; Herbert, Pouquet \& Marino 2014). We will therefore not consider the constraints related to the conservation of Ertel potential vorticity in the remaining of this paper. In the context of equilibrium statistical mechanics, this amounts to assuming that the entropy maxima obtained with and without these constraints are the same.

\subsection{Microscopic configurations, macroscopic description and variational problem}

For an isolated system, the fundamental postulate of equilibrium statistical mechanics is the equiprobability of the microscopic configurations corresponding to the same values of the dynamical invariants.

The first step is to define what are the relevant phase-space variables describing these microscopic configurations. Those variables must satisfy Liouville's theorem, which means that the flow in phase space is non-divergent. This ensures that microscopic configurations remain equiprobable during the time evolution of the system. We show in appendix A that the quadruplet of fields $(b, \boldsymbol{u})$ satisfy such a Liouville theorem, and are therefore relevant phase-space variables.

The second step is to identify the relevant dynamical invariants, which are here the total energy and the global distribution of buoyancy levels, defined in (2.6) and in (2.4), respectively. The ensemble of microscopic configurations characterised by the same dynamical invariants is called the microcanonical ensemble. This is the relevant 
ensemble to consider for an isolated system such as the unforced, inviscid, adiabatic Boussinesq system.

The third step is to identify relevant macrostates, which describe an ensemble of microscopic configurations. We introduce for that purpose the probability $\rho(\boldsymbol{x}, \sigma, \boldsymbol{v})$ of finding the buoyancy level $\sigma$ and the velocity level $\boldsymbol{v}$ in the vicinity of point $\boldsymbol{x}$. It is normalised at each point:

$$
\forall \boldsymbol{x} \in \mathcal{V}_{\boldsymbol{x}}, \quad \mathcal{N}_{x}[\rho]=\int_{\mathcal{V}_{\boldsymbol{v}}} \mathrm{d} \boldsymbol{v} \int_{\mathcal{V}_{\sigma}} \mathrm{d} \sigma \rho(\boldsymbol{x}, \sigma, \boldsymbol{v})=1,
$$

where the integral bounds are

$$
\mathcal{V}_{v}=[-\infty,+\infty]^{3}, \quad \mathcal{V}_{\sigma}=[-\infty,+\infty]
$$

Each microscopic state $(b(\boldsymbol{x}), \boldsymbol{u}(\boldsymbol{x}))$ is described at a macroscopic level by the probability distribution function (PDF) $\rho(\boldsymbol{x}, \sigma, \boldsymbol{v})$, and many microscopic configurations are in general associated with a given PDF $\rho(\boldsymbol{x}, \sigma, \boldsymbol{v})$, which is called a Young measure in mathematics; see e.g. Robert \& Sommeria (1991).

Let us define more precisely how to compute the macroscopic state $\rho(\boldsymbol{x}, \sigma, \boldsymbol{v})$ from a given microscopic configuration $(b(\boldsymbol{x}), \boldsymbol{u}(\boldsymbol{x}))$, which will be useful to count the number of microscopic configurations associated with a given macrostate. For that purpose, we follow a procedure which is standard in the framework of equilibrium statistical mechanics of fluid systems, using a discrete approximation of the continuous fields. We consider a uniform coarse-grained grid containing $N$ macrocells, and a fine-grained grid obtained by dividing each macrocell of the coarse-grained grid into a uniform grid containing $M$ fluid particles, see figure 1 . On the one hand, discretisation of the microscopic field $b(\boldsymbol{x})$ and $\boldsymbol{u}(\boldsymbol{x})$ are defined on the fine-grained grid, which contains $M N$ fluid particles. This procedure also requires a discretisation of the buoyancy and velocity levels carried by the fluid particles, which is further discussed in appendix B. On the other hand, the discrete approximation of the PDF $\rho$ is defined on the coarse-grained grid.

For a given microscopic configuration, one can compute within each macrocell of the coarse-grained grid the frequency of occurrence of buoyancy and velocity levels (a normalised histogram). In the limit $M \rightarrow+\infty$, for a prescribed value of $N$, the discrete approximations of the microscopic configurations tend to the continuous ones, and the discrete approximation of the PDF $\rho$ is equivalent to the frequency of occurrence of buoyancy and velocity levels within each macrocell. In other words, the discrete approximation of the PDF $\rho$ can be interpreted as the volume proportion of fluid particles carrying the buoyancy level $\sigma$ and velocity level $\boldsymbol{v}$ inside each macrocell. The continuous PDF field $\rho$ is then recovered by considering the limit $N \rightarrow+\infty$, which corresponds to the limit of infinitesimal macrocells. Several useful macroscopic quantities can be deduced from $\rho$, such as the macroscopic buoyancy field

$$
\bar{b}(\boldsymbol{x})=\int_{\mathcal{V}_{\sigma}} \mathrm{d} \sigma \int_{\mathcal{V}_{v}} \mathrm{~d} \boldsymbol{v} \rho \sigma,
$$

and the local eddy kinetic energy field

$$
\frac{1}{2} \overline{\boldsymbol{u}^{2}}(\boldsymbol{x})=\int_{\mathcal{V}_{\sigma}} \mathrm{d} \sigma \int_{\mathcal{V}_{v}} \mathrm{~d} \boldsymbol{v} \frac{1}{2} \rho \boldsymbol{v}^{2} .
$$



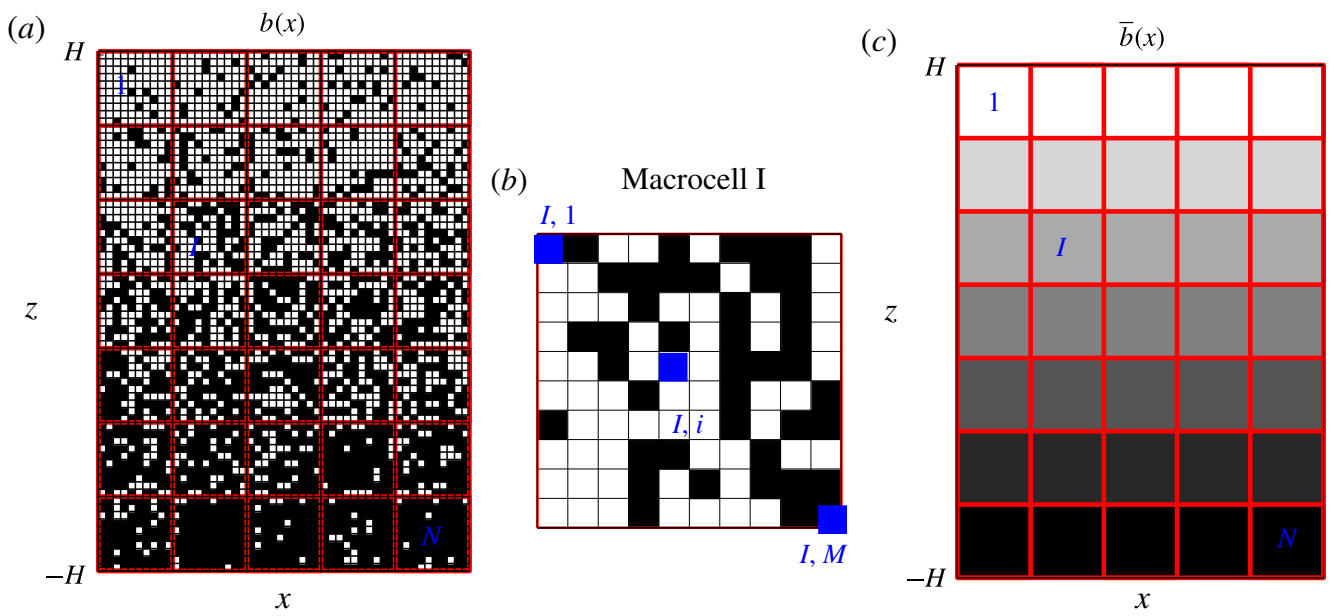

FIGURE 1. (Colour online) (a) A microscopic configuration of the discretised buoyancy field $b(\boldsymbol{x})$. The discretised buoyancy field is defined on a uniform fine-grained grid containing $M \times N$ elements, where $N$ is the number of grid points of the uniform coarse-grained grid (red colour). (b) Zoom on a single macrocell, containing $M$ microcells. Each microcell contains one fluid particle. Here we consider the case of a two-level system: the buoyancy carried by each fluid particle is $b= \pm \Delta b / 2$. (c) The macroscopic buoyancy field $\vec{b}(x)$ is defined on the uniform coarse-grained grid (red colour), and is computed in the limit $M \rightarrow+\infty$ by averaging the microscopic buoyancy field within each macrocell, see e.g. Miller (1990), Tabak \& Tal (2004).

Within the framework of the discrete approximation depicted in figure 1, those macroscopic quantities correspond to averages over macrocells, i.e. to a spatial coarse graining at the scale of a macrocell $\sim N^{-1 / 3}$. Importantly, the small-scale fluctuations described by the macroscopic states are confined at spatial scales below this coarse-graining scale, which tends to zero in the limit $N \rightarrow+\infty$.

The advantage of considering the probability field $\rho$ rather than only the coarsegrained fields such as $\bar{b}$ for a macroscopic description of the system is that global constraints provided by dynamical invariants can be expressed in terms of $\rho$. The global constraints are given by the energy and the global distribution of buoyancy levels, which are defined as functional of phase-space variables $(\boldsymbol{u}, b)$ in $(2.6)$ and (2.4), respectively. Considering the discrete approximation described in the previous paragraph, decomposing the spatial integrals appearing in (2.6) and (2.4) as a sum of spatial integrals over each macrocells, remembering then that the PDF $\rho$ is the frequency of occurrence of buoyancy and velocity levels within a given macrocell, and taking finally the limit $M \rightarrow+\infty, N \rightarrow+\infty$, the energy and the global distribution of buoyancy levels can be expressed as functionals of the PDF $\rho$ :

$$
\begin{gathered}
\mathcal{E}[\rho]=\int_{\mathcal{V}_{x}} \mathrm{~d} \boldsymbol{x} \int_{\mathcal{V}_{v}} \mathrm{~d} \boldsymbol{v} \int_{\mathcal{V}_{\sigma}} \mathrm{d} \sigma \rho\left(\frac{\boldsymbol{v}^{2}}{2}-\sigma z\right)+\int_{\mathcal{V}_{\boldsymbol{x}}} \mathrm{d} \boldsymbol{x} z b_{s}, \\
\mathcal{G}_{\sigma}[\rho]=\int_{\mathcal{V}_{\boldsymbol{x}}} \mathrm{d} \boldsymbol{x} \int_{\mathcal{V}_{\boldsymbol{v}}} \mathrm{d} \boldsymbol{v} \rho .
\end{gathered}
$$

The microcanonical ensemble is defined by the ensemble of microstates characterised by the same energy $E$ and global distribution of buoyancy levels $G(\sigma)$. This ensemble 
contains therefore all the macroscopic states that satisfy the dynamical constraints $\mathcal{E}[\rho]=E$ and $\mathcal{G}_{\sigma}[\rho]=G(\sigma)$.

The last step is to count how many microscopic configurations are associated with a given macrostate. Considering our discrete approximation of the fields, it is shown in appendix B that within the microcanonical ensemble, an overwhelming number of the microscopic configurations is concentrated close to the most probable macrostate, which maximises the macrostate entropy

$$
\mathcal{S}=-\int_{\mathcal{V}_{x}} \mathrm{~d} \boldsymbol{x} \int_{\mathcal{V}_{v}} \mathrm{~d} \boldsymbol{v} \int_{\mathcal{V}_{\sigma}} \mathrm{d} \sigma \rho \log \rho .
$$

The expression of the macrostate entropy given in (2.13) is a classical one, especially in the context of two-dimensional turbulence (Miller 1990; Robert \& Sommeria 1991). A rigorous derivation of such macrostate entropy requires the use of large deviation theory; see e.g. Touchette (2009) for an introduction to those tools. A key difficulty in deriving rigorously this macrostate entropy from the usual Boltzmann entropy is that the microstates are continuous fields which contain an infinite number of degrees of freedom, and which are constrained by an infinite number of dynamical invariants. Several discretisation procedures have been proposed to bypass this difficulty, see e.g. Michel \& Robert (1994), Boucher, Ellis \& Turkington (2000), Bouchet \& Corvellec (2010), Potters et al. (2013), Renaud et al. (2016). A similar formula has been derived previously by Tabak \& Tal (2004) in the context of non-rotating, density stratified Boussinesq fluids, in the particular case of a two-level buoyancy configuration. Here we have generalised this result to arbitrary buoyancy distribution, and more importantly, we have included the velocity field in the description of the microstate, which is essential to account for energy conservation.

\subsection{Computation of the most probable macrostate and general properties of the equilibrium states}

The first step to find the equilibrium state is to compute critical points of the variational problem given by the equilibrium theory, i.e. to find the field $\rho$ such that first variations of the macrostate entropy (2.13) around this state vanish, given the constraints of the problem given by $\mathcal{E}[\rho]=E, \mathcal{G}_{\sigma}[\rho]=G(\sigma), \mathcal{N}_{x}[\rho]=1$, where $\mathcal{E}$ is the energy defined in (2.11), $\mathcal{G}_{\sigma}$ is the global distribution of buoyancy defined in (2.12) and $\mathcal{N}_{x}$ the local normalization of the PDF expressed in (2.7). One needs for that purpose to introduce the Lagrange multipliers $\beta_{t}, \gamma(\sigma), \xi(\boldsymbol{x})$ associated with those constraints. Computing first variations with respect to the probability field $\rho$ yields

$$
\delta \mathcal{S}-\beta_{t} \delta \mathcal{E}+\int_{\mathcal{V}_{\sigma}} \mathrm{d} \sigma \gamma(\sigma) \delta \mathcal{G}_{\sigma}+\int_{\mathcal{V}_{x}} \mathrm{~d} \boldsymbol{x} \xi(\boldsymbol{x}) \delta \mathcal{N}_{x}=0 .
$$

Using the expression of the entropy, of the energy, of the global distribution of buoyancy and of the normalisation constraints given respectively in (2.13), (2.11), (2.12) and (2.7), (2.14) yields

$$
\int_{\mathcal{V}_{x}} \mathrm{~d} \boldsymbol{x} \int_{\mathcal{V}_{v}} \mathrm{~d} \boldsymbol{v} \int_{\mathcal{V}_{\sigma}} \mathrm{d} \sigma\left((1+\log \rho)+\beta_{t}\left(\frac{\boldsymbol{v}^{2}}{2}-\sigma z\right)-\gamma(\sigma)-\xi(\boldsymbol{x})\right) \delta \rho=0 .
$$


This equality is true for any $\delta \rho$, which, using the normalisation constraint in (2.7), yields the following necessary and sufficient condition for $\rho$ to be a critical point of the variational problem:

$$
\rho(\boldsymbol{x}, \sigma, \boldsymbol{v})=\left(\frac{\beta_{t}}{2 \pi}\right)^{3 / 2} \mathrm{e}^{-\beta_{t}\left(\boldsymbol{v}^{2} / 2\right)} \rho_{b}(z, \sigma),
$$

with

$$
\rho_{b}(z, \sigma) \equiv \frac{\mathrm{e}^{\beta_{t} \sigma z+\gamma(\sigma)}}{\mathcal{Z}(z)}, \quad \mathcal{Z}(z) \equiv \int_{\mathcal{V}_{\sigma}} \mathrm{d} \sigma \mathrm{e}^{\beta_{t} \sigma z+\gamma(\sigma)} .
$$

The values of the Lagrange multipliers $\beta_{t}$ and $\gamma(\sigma)$ are implicitly determined by the expression of the constraints $\mathcal{E}[\rho]=E$ and $\mathcal{G}_{\sigma}[\rho]=G(\sigma)$, given by (2.11) and (2.12), respectively.

The probability density field (2.16) is expressed as a product of the probabilities for buoyancy and velocity, which means that $b$ and $\boldsymbol{u}$ are two independent quantities at equilibrium. The predicted velocity distribution is Gaussian, with zero mean $(\overline{\boldsymbol{u}}=0)$, isotropic and homogeneous in space. It is therefore fully characterised by the local eddy kinetic energy

$$
e_{c} \equiv \frac{1}{2} \overline{u^{2}}=\frac{3}{2} \frac{1}{\beta_{t}} .
$$

The inverse of $\beta_{t}$ defines an effective 'temperature' of the turbulent field, corresponding to the turbulent agitation of fluid particles. Remarkably, the three-dimensional nature of the flow appears only in this equation and nowhere else. A two-dimensional case would just have a different relation between kinetic energy and this effective temperature.

The predicted buoyancy distribution $\rho_{b}$ depends only on the height coordinate $z$. The equilibrium theory predicts therefore that the local fluctuations of buoyancy are invariant in the horizontal. It means that in the remaining of this paper, the quantities - can be interpreted either as a local coarse graining or as a horizontal average. Similarly, the quantity $\rho_{b}$ can be interpreted either as a local distribution of buoyancy or as the distribution of buoyancy over an horizontal plane.

Equation (2.17) relates the mean buoyancy profile and its fluctuations to the effective turbulent temperature. Buoyancy moments are defined at each height in terms of $\rho_{b}(z, t)$ as

$$
\overline{b^{n}}(\boldsymbol{x}) \equiv \int_{\mathcal{V}_{\sigma}} \mathrm{d} \sigma \sigma^{n} \rho_{b} .
$$

From (2.17) we get the relations

$$
\bar{b}=\frac{1}{\beta_{t}} \frac{\mathrm{d} \log \mathcal{Z}}{\mathrm{d} z}, \quad \overline{b^{2}}-\bar{b}^{2}=\frac{1}{\beta_{t}^{2}} \frac{\mathrm{d}^{2} \log \mathcal{Z}}{\mathrm{d} z^{2}} .
$$

Using those expressions and (2.18), one gets finally an expression relating the mean buoyancy profile to the ratio of the buoyancy fluctuations to the kinetic energy fluctuations:

$$
\frac{\mathrm{d} \bar{b}}{\mathrm{~d} z}=3 \frac{\overline{b^{2}}-\bar{b}^{2}}{2 e_{c}} .
$$


In the case of a strong stratification, the local variance of buoyancy is proportional to the small vertical displacement of fluid elements, so this relation can be interpreted as an equipartition between kinetic and potential energy fluctuations, as further discussed in $\S 4.3$.

The equilibrium state has a peculiar spatial structure: the buoyancy field $b$ is characterised by a smooth coarse-grained buoyancy profile $\bar{b}(z)$ superimposed with small-scale buoyancy fluctuations. More precisely, the theory predicts that when performing a local coarse graining of the microscopic buoyancy and velocity fields at a scale $l$ (the scale of the macrocell within the framework of our discrete model depicted in figure 1), the small-scale fluctuations are confined at scales smaller than the coarse-graining scale $l$, no matter how small the coarse-graining length scale $l$.

In the case of a decaying experiment with weak molecular viscosity and diffusivity, the subgrid-scale velocity fluctuations of the equilibrium state correspond to the amount of kinetic energy that will be dissipated by viscosity during the whole decay, the subgrid-scale buoyancy fluctuations of the equilibrium state correspond to the amount of buoyancy fluctuations locally dissipated by diffusivity during the whole decay. As a result, the equilibrium state $\bar{b}$ corresponds to the background buoyancy profile that will be measured after a mixing event, once the system has reached a state of rest in a decaying experiment. The underlying hypothesis is that the system reaches the equilibrium states before molecular effects become important.

\section{Computation of mean equilibrium buoyancy profiles}

\subsection{The two-level case}

We discussed in the previous subsection the general case with a continuum of buoyancy levels. In the particular case with a finite number of buoyancy levels (say $K$ levels $\sigma_{k}$ with $1 \leqslant k \leqslant K$ ), the buoyancy field is described at a macroscopic level by $p_{k}(\boldsymbol{x})$, which is the probability of measuring the level $\sigma_{k}$ at point $\boldsymbol{x}$ with $\sum_{k=1}^{K} p_{k}(\boldsymbol{x})=1$, see appendix B. The same arguments as in $\S 2.3$ for the computation of the equilibrium state then yield

$$
p_{k}(z) \equiv \frac{\mathrm{e}^{\beta_{t} \sigma_{k} z+\gamma_{k}}}{\sum_{k=1}^{K} \mathrm{e}^{\beta_{t} \sigma_{k} z+\gamma_{k}}}, \quad \beta_{t}=\frac{3}{2 e_{c}},
$$

where the values of the Lagrange multipliers $\beta_{t}$ and $\left\{\gamma_{k}\right\}_{1 \leqslant k \leqslant K}$ are implicitly determined by the energy constraint and conservation of the total volume occupied by each buoyancy level $\sigma_{k}$.

Let us restrict ourselves to the case of an initial state composed of two buoyancy levels in equal proportion with

$$
\forall x \in \mathcal{V}_{x}, \quad b(x) \in\left\{-\frac{\Delta b}{2}, \frac{\Delta b}{2}\right\} .
$$

The only dimensionless parameter of the problem within the statistical mechanics framework is given by the global Richardson number based on the total height $2 \mathrm{H}$, buoyancy jump $\Delta b$ and square of velocity fluctuations $2 e_{c}$ :

$$
R i \equiv \frac{H \Delta b}{e_{c}} .
$$


This global Richardson number based on the domain height $H$ is different from the bulk Richardson number $R i_{b}=\Delta b L_{t} / e_{c}=\left(L_{t} / H\right) R i$ based on the turbulent length scale $L_{t}$, which is commonly used in the context of turbulent mixing in stratified fluids; see e.g. Fernando (1991). The statistical mechanics prediction depends only on the total energy, not on its injection scale $L_{t}$. This point will be further discussed in $\S 4.4$.

We denote $p_{+}(z)$ the probability of measuring $\Delta b / 2$ at height $z$. According to the notation used in (3.1), we get $\sigma_{1}=-\Delta b / 2, \sigma_{2}=\Delta b / 2, p_{1}=1-p_{+}, p_{2}=p_{+}$, with

$$
p_{+}(z)=\frac{\mathrm{e}^{(3 R i / 4)(z / H)}}{\mathrm{e}^{-(3 R i / 4)(z / H)}+\mathrm{e}^{(3 R i / 4)(z / H)}},
$$

where we have used the symmetry with respect to $z=0(p+(z)=-p(-z))$ and the fact that the two buoyancy levels are in equal proportions $\left(\int_{-H}^{+H} \mathrm{~d} z p_{+}=\int_{-H}^{+H} \mathrm{~d} z p_{-}\right)$to eliminate the Lagrange parameters $\gamma_{1}, \gamma_{2}$ in (3.1).

Equation (3.4) is reminiscent of the Fermi-Dirac distribution. Indeed, the conservation of buoyancy plays here the same role as the exclusion principle for the statistics of fermions: within the framework of the discretised model depicted in figure 1, the buoyancy carried by a fluid particle at a given grid point can only take one value among $-\Delta b / 2$ and $\Delta b / 2$. Following this analogy, the buoyancy field is a collection of fluid particles carrying the potential energy $e_{p}= \pm 1 / 2 z \Delta b$, with a Fermi level $\varepsilon_{f}=0$, in thermal contact with a heat bath characterised by the inverse temperature $\beta_{t}$.

Using (3.4) and (2.18), the mean density profile $\bar{b}=(\Delta b / 2) p_{+}-\Delta b / 2\left(1-p_{+}\right)$is expressed as

$$
\bar{b}(z)=\frac{\Delta b}{2} \tanh \left(\frac{3 R i}{4} \frac{z}{H}\right) .
$$

Large global Richardson numbers $R i \gg 1$ correspond to sharp interfaces: the kinetic energy is too small to allow for large excursion of fluid particles away from the rest position. By contrast, small global Richardson numbers $R i \ll 1$ correspond to a homogenised buoyancy field: the total kinetic energy is much larger than the energy required to mix the buoyancy field. This tanh profile was previously obtained by Tabak $\&$ Tal (2004) using similar arguments, but without relating the effective temperature to the kinetic energy of the flow in a consistent theory. Our approach allows for a direct interpretation of the effective temperature of the flow as the local turbulent kinetic energy, which will make possible quantitative estimate for mixing efficiency.

\subsection{A relaxation equation towards the equilibrium states}

The expression for the equilibrium state given in (2.17) requires the knowledge of the Lagrange multipliers $\gamma(\sigma)$ and $e_{c}$, which depend implicitly on the constraints $G(\sigma)$ and $E$. This makes analytical computations of those equilibria very challenging. Solutions may be obtained in particular cases, such as for the two-level configuration analysed in $\S 3.1$, but more generally it must be determined numerically.

We devise for that purpose an algorithm based on a maximum entropy production principle, which was introduced by Robert \& Sommeria (1992) in order to compute equilibrium states of two-dimensional Euler flows. The idea of the algorithm is to consider a time-dependent probability distribution function

$$
\rho(\sigma, \boldsymbol{x}, \boldsymbol{v}, t)=\left(\frac{3}{4 \pi e_{c}(t)}\right)^{3 / 2} \mathrm{e}^{-\left(3 / 2 e_{c}(t)\right)\left(\boldsymbol{v}^{2} / 2\right)} \rho_{b}(z, \sigma, t),
$$


where the PDF $\rho_{b}(z, \sigma, t)$ and the local kinetic energy $e_{c}(t)$ depend on time, and can be different from the pdf and the kinetic energy of the actual equilibrium state. We derive in appendix $\mathrm{C}$ a dynamical equation for $\rho_{b}$ that conserves the total energy and the global distribution of buoyancy levels, while maximising the entropy production at each time:

$$
\partial_{t} \rho_{b}=\partial_{z}\left[D\left(\partial_{z} \rho_{b}-\frac{3}{2 e_{c}}(\sigma-\bar{b}) \rho_{b}\right)\right],
$$

where $D$ is an arbitrary positive diffusion coefficient. The kinetic energy $e_{c}$ defined in (2.18) is expressed in terms of the total energy $E$ and the buoyancy profile $\bar{b}(z, t)$ by using (2.11):

$$
e_{c}=\frac{E}{V}+\frac{1}{2 H} \int_{-H}^{+H} \mathrm{~d} z\left(\bar{b}-b_{s}\right) z
$$

with $V$ the volume of the flow domain.

Maximising the entropy production ensures that the system relaxes towards an equilibrium state. Indeed, using (2.17)-(2.18) and the first equality in (2.20), the equilibrium states can be written as

$$
\rho_{b}(\sigma, z)=\rho_{b}(\sigma, 0) \mathrm{e}^{\left(3 / 2 e_{c}\right)\left(\sigma z-\int_{0}^{z} \mathrm{~d} z^{\prime} \bar{b}\left(z^{\prime}\right)\right)},
$$

which is also the expression of any stationary solution of (3.7). According to equation (3.7) the equilibrium state can be interpreted as the result of a compensation between usual turbulent diffusion and a drift term corresponding to restratification of buoyancy fluctuations. We stress that the convergence towards equilibrium depends on the parameter $D$, but that the equilibrium itself does not depend on this parameter. This is why it can be chosen arbitrarily.

Assuming that the initial energy $E$ injected into the system and that the background buoyancy profile $b_{s}(z)$ are known, one can then use the relaxation algorithm (3.7), starting from the state

$$
\rho_{b}(z, \sigma, 0)=\delta\left(b_{s}(z)-\sigma\right), \quad e_{c}(0)=\frac{E}{V}
$$

Equation (3.7) is an integro-differential equation, because the local kinetic energy is a functional of the macroscopic vertical buoyancy profile. Its numerical implementation is much easier assuming that $e_{c}$ is a constant. One then loses energy conservation, but the equation still conserves the global buoyancy distribution, assuming no buoyancy fluxes at the upper and lower boundaries. It can be shown that this process minimises the free-energy production defined as $\dot{\mathcal{F}}=-\dot{\mathcal{S}}+\beta_{t} \dot{\mathcal{E}}$, where the upper dot stands for a time derivative, and where $\beta_{t}=3 /\left(2 e_{c}\right)$ can be interpreted as the inverse of an effective turbulent temperature. Indeed, assuming constant local kinetic energy amounts to a computation of the equilibrium state within the canonical ensemble where the 'heat bath' is provided by turbulent agitation. In order to solve numerically (3.10) with constant $e_{c}$, we first assume a discretisation of the global buoyancy distribution into $N_{\sigma}$ buoyancy levels denoted $\sigma_{n}$ with $1 \leqslant n \leqslant N_{\sigma}$. Denoting $\rho_{b, n}(z, t)$ the probability to measure the level $\sigma_{n}$ in the vicinity of height $z$ at time $t$, we obtain a system of onedimensional parabolic partial differential equations for $\left\{\rho_{b, n}(z, t)\right\}_{1 \leqslant n \leqslant N_{\sigma}}$, which can be solved using standard numerical procedures. This dynamical system is integrated in 

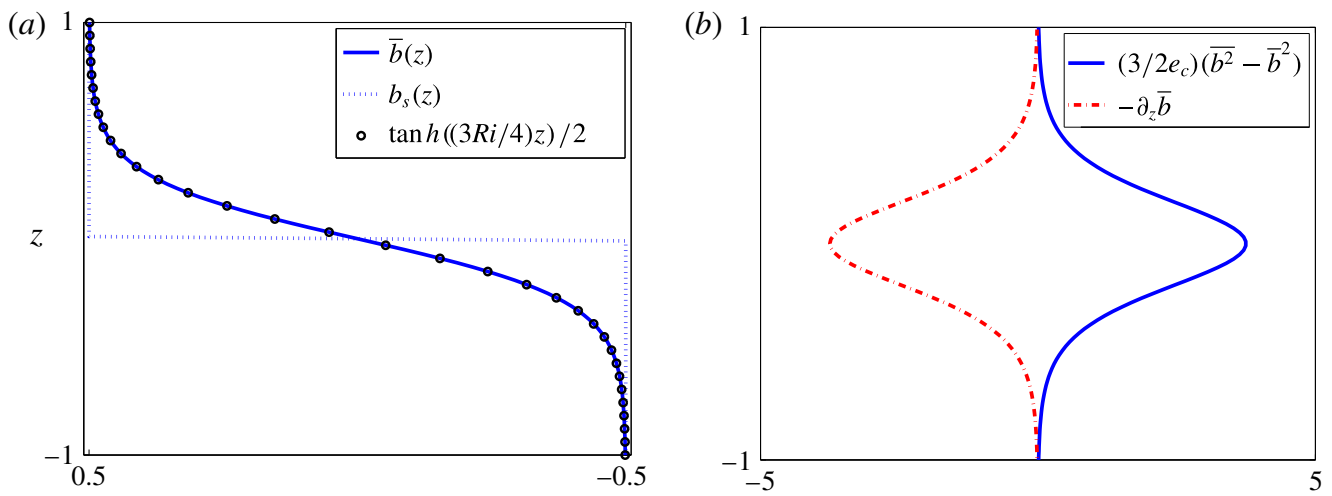

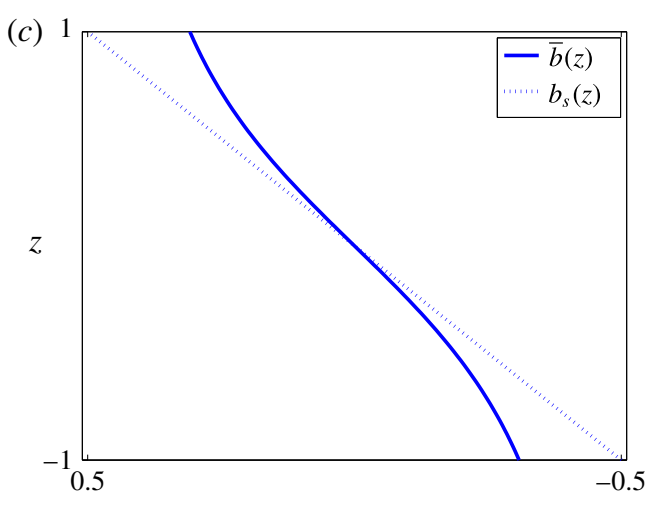

Buoyancy

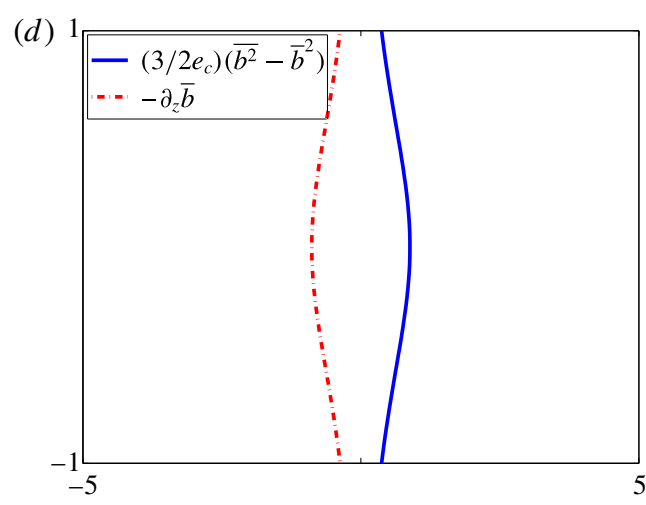

Buoyancy flux

FIgURE 2. (Colour online) (a) Plain blue line: equilibrium state $\bar{b}(z)$ computed numerically in the case $R i=10$, where $R i=H \Delta b / e_{c}$ is the global Richardson number. Here $H=1, \Delta b=1$. The dotted blue line: corresponding background buoyancy profile $b_{s}(z)$ (here a two-layer case). Black circles: analytical expression from (3.5) for the equilibrium state of the two-level system. The buoyancy increases from right to left on the horizontal axis. (b) Compensation of the downgradient buoyancy flux with the restratification term proportional to buoyancy fluctuations (with $D=1$ ). The total buoyancy is the sum of those two terms, which is zero at equilibrium. $(c, d)$ Same as $(a, b)$ in the case of an initial linear background buoyancy profile (no analytical predictions in that case).

time until a steady state is reached. This steady state is the equilibrium state. Once the equilibrium state associated with a given value of $e_{c}$ is computed, it is straightforward to compute its total energy $E$ using (2.11). One can then check that varying $e_{c}$ from 0 to $+\infty$ amounts to varying $E$ from 0 to $+\infty$. This procedure therefore provides the complete set of equilibria associated with any given background buoyancy profile.

We show in figure 2 two examples of equilibrium states computed by this procedure, assuming no buoyancy fluxes at the upper and lower boundaries. Panels $(a, b)$ correspond to the two-level configuration. As expected from (3.5), the mean equilibrium buoyancy profile is characterised by a tanh shape in that case. Panel $(b)$ confirms that this equilibrium state may be interpreted as the result of a balance between a classical downgradient term $-D \partial_{z} \bar{b}$ modelling turbulent transport and a term $D\left(3 / 2 e_{c}\right)\left(\overline{b^{2}}-\bar{b}^{2}\right)$ modelling restratification. 
Panels $(c, d)$ correspond to the more complicated case of a linear profile for the background buoyancy profile, for which no analytical results exist. Just as in the twolayer case, we see enhanced buoyancy fluctuations in the domain bulk. This numerical method can easily be applied to any background buoyancy profile, and will be applied in next section to the computation of mixing efficiency.

\section{Computation of mixing efficiency in decaying flows}

\subsection{Irreversibility and mixing efficiency}

We argue in the following that the computation of the equilibrium states for the inviscid, adiabatic system can be used to obtain quantitative predictions for the efficiency of mixing in decaying stratified turbulence.

The first assumption is that molecular viscosity and diffusivity only play a secondary role in the limit of large Reynolds and Péclet numbers. More precisely, we assume that the time scale to reach the equilibrium state of the inviscid, adiabatic dynamics is smaller than the typical time scale of dissipative effects. In other words, inertial dynamics governs the amount of small-scale velocity and buoyancy fluctuations that are created on a short time scale, and the only effect of viscosity and diffusivity is to smooth out these fluctuations on a longer time scale.

The second assumption is that the flow system evenly explores phase space through turbulent stirring, which is necessary to use statistical mechanics predictions. According to the theory, the macroscopic buoyancy profile $\bar{b}$ and the local distribution of small-scale fluctuations do not evolve in time anymore once the equilibrium state is reached: the equilibrium state is an attractor for the dynamics. In that respect, the purely inertial, inviscid and adiabatic dynamics is irreversible. In other words, even if the process described by the equilibrium theory is pure stirring, it implies irreversible mixing of the buoyancy field at a coarse-grained level. Assuming that this stationary property of $\bar{b}$ persists in the presence of weak viscosity and weak dissipation, we see from (2.21) that the rate of local small-scale kinetic energy dissipation $\mathrm{d} \log e_{c} / \mathrm{d} t$ should be equal to the rate of dissipation for the local variance of local buoyancy fluctuations $\mathrm{d} \log \left(\overline{b^{2}}-\bar{b}^{2}\right) / \mathrm{d} t$.

Let us assume that a given amount of energy denoted $E_{i n j}$ is injected in a fluid initially at rest, characterised by a background buoyancy profile $b_{s}(z)$. The injected energy may either be purely kinetic (through mechanical stirring) or purely potential (for instance by turning the tank upside down into an unstable configuration). Once the equilibrium state is reached, part of this energy is carried by small-scale velocity fluctuations, and the remaining part is used to maintain the potential energy of the system at a higher value than the potential energy of the background state. The transfer of part of the initial energy present at a coarse-grained level into subgrid-scale (fine-grained) fluctuations is very much similar to the effect of viscosity, which transfers energy from the degrees of freedom of the fluid motion to these of thermal fluctuations.

The total kinetic energy carried by the equilibrium state is denoted $E_{c}=V e_{c}$ with $e_{c}$ the local kinetic energy density, homogeneous in space. This kinetic energy takes the form of small-scale fluctuations, that will be eventually dissipated in a decaying experiment with weak viscosity and the quantity $E_{c}$ can then be interpreted as the temporal integral of viscous dissipation.

Turbulent stirring implies rearrangements of fluid parcels, and such rearrangements from $b_{s}(z)$ to $b(x, y, z)$ are necessarily associated with an increase of potential energy

$$
E_{p}=-\int_{\mathcal{V}_{x}} \mathrm{~d} \boldsymbol{x}\left(b-b_{s}\right) z .
$$


At equilibrium, this quantity can be expressed in terms of the macroscopic buoyancy profile $\bar{b}$ which depends only on $z$ :

$$
E_{p}=-\frac{V}{2 H} \int_{-H}^{+H} \mathrm{~d} z\left(\bar{b}-b_{s}\right) z
$$

This definition is equivalent to the classical definition of the available potential energy. However, as explained above, the convergence towards the equilibrium buoyancy profile is irreversible. Once the equilibrium is reached, the available potential energy $E_{p}$ has been irreversibly transferred to smaller scales, and cannot be transferred anymore into another form of energy. It would inescapably result into molecular mixing in the presence of molecular diffusion. In that case, $E_{p}$ would corresponds to the increase of the background potential energy, which is consistent with Winters et al. (1995).

We define the mixing efficiency as

$$
\eta \equiv \frac{E_{p}}{E_{p}+E_{c}}
$$

where $E_{p}+E_{c}=E_{i n j}$ is the total energy injected into the system. This definition of mixing efficiency is bounded between 0 and 1 . Since $E_{c}$ is the total amount of kinetic energy lost at small scale, and since $E_{p}$ corresponds to an irreversible increase of potential energy according to statistical mechanics theory, our definition of $\eta$ is equivalent to the long time limit of the cumulative mixing efficiency (Peltier \& Caulfield 2003), or to the integrated flux Richardson number (Linden 1979).

We stress finally that the equilibrium theory does not predict a temporal evolution for the system but just the final outcome of turbulent stirring under the assumption of random evolution without forcing and dissipation. It provides therefore a global (integrated over the whole domain) and cumulative (integrated over sufficiently large time) prediction for the efficiency of mixing.

\subsection{Numerical computation in the general case}

We show in figure 3 how the mixing efficiency $\eta$ varies with the global Richardson number $R i=H \Delta b / e_{c}$, with $\Delta b=b_{s}(H)-b_{s}(-H)$. We consider two different buoyancy profiles $b_{s}(z)$ : case $(a)$ is the two-level configuration corresponding to a background profile with two homogeneous layers of equal depth, for which an analytical solution exits; case (b) corresponds to a linear background buoyancy profile. Considering those two cases allows us to show very different behaviour for the variations of mixing efficiency as a function of the Richardson number $R i$.

The kinetic energy $e_{c}$ appearing in the Richardson number is not a control parameter, but one can check a posteriori that $E_{c}=V e_{c}$ is always of the same order of magnitude as the injected energy $E_{i n j}$, which is a control parameter. In a direct numerical simulations with non-zero viscosity, $E_{c}$ would be the actual amount of kinetic energy dissipated during the turbulent decay.

We see in figure 3 that whatever the background buoyancy profile, the equilibrium buoyancy profile $\bar{b}$ can be considered as almost completely homogenised in the low Richardson number limit $R i \ll 1$. In that case, most of the injected energy is lost in small-scale velocity fluctuations with $E_{c}=E_{i n j}$ and the fluid is well mixed, so that $\bar{b}$ 

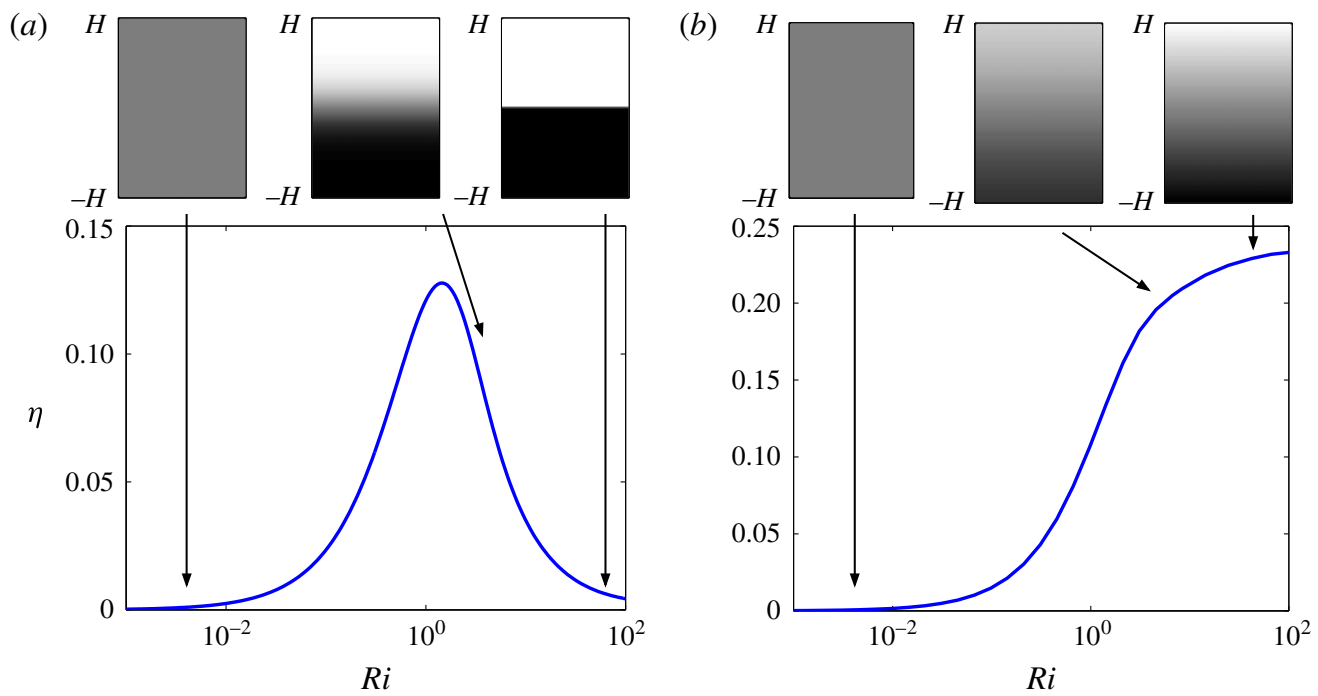

FIgURE 3. (Colour online) Variation of the mixing efficiency $\eta=E_{p} / E_{i n j}$ with the Richardson number $R i=H \Delta b / e_{c}$ (a) for a background buoyancy profile with two homogeneous layers, $(b)$ for an initial linear background buoyancy profile. The three insets show the equilibrium buoyancy field $\bar{b}$ for $R i=0.07,7,70$.

is a constant, and (4.2) reduces to $E_{p}=(V / 2 H) \int_{-H}^{+H} b_{s} z \mathrm{~d} z$. The mixing efficiency is then given by

$$
\eta={ }_{R i \ll 1} R i \Xi\left[b_{s}\right] \quad \text { with } \Xi\left[b_{s}\right] \equiv \frac{1}{2 \Delta b H^{2}} \int_{-H}^{+H} b_{s} z \mathrm{~d} z \text { when } R i \ll 1 .
$$

The numerical coefficient $\Xi\left[b_{s}\right]$ is bounded in [0 1] and characteristic of the shape of the background buoyancy profile, hence of the distribution of available densities. It is equal to 0 for a homogeneous fluid, $1 / 6$ for a linear stratification and $1 / 4$ for a two-layer system. Whatever this background buoyancy profile, the mixing efficiency scales linearly with the Richardson number is the limit of weak Richardson numbers.

By contrast, we see in figure 3 that the large Richardson number behaviour of the mixing efficiency depends drastically on the background buoyancy profile $b_{s}$ : the mixing efficiency decreases to zero with increasing Richardson numbers in the twolevel case of figure $3(a)$, while it increases to an asymptotic value close to 0.25 in the linearly stratified case of figure $3(b)$. We show analytically in the next subsection that an asymptotic value of $\eta=0.25$ is indeed expected in a low energy limit, as a consequence of energy equipartition, provided that the stratification of the background profile is always strictly positive $\left(\partial_{z} b_{s}>0\right.$ for $\left.-H \leqslant z \leqslant H\right)$.

\subsection{Energy equipartition and mixing efficiency for high Richardson numbers}

The potential energy $E_{p}$ defined in (4.1) is a linear functional of $b-b_{s}$, which is a priori sign indefinite. However, the conservation of the global distribution of buoyancy levels (prescribed by $b_{s}$ ) provides a strong constraint on admissible buoyancy levels $b$, and hence on admissible values for $E_{p}$. A direct consequence of these conservation laws it that the potential energy is strictly positive unless $b=b_{s}$. 
Denoting $Z_{s}\left(b_{s}\right)$ the height of fluid particles carrying buoyancy level $b_{s}$ in the background buoyancy profile, using an asymptotic expansion in terms of $b-b_{s}$ and assuming $\mathrm{d} b_{s} / \mathrm{d} z=b_{s}^{\prime}>0$, one can use the conservation laws related to buoyancy (Casimir functionals) to obtain an explicit quadratic form for the potential energy in a weak energy limit (Shepherd 1993):

$$
E_{p}=\frac{1}{2} \int_{\mathcal{V}_{x}} \mathrm{~d} x \frac{\left(b-b_{s}\right)^{2}}{b_{s}^{\prime}}+O\left(Z_{s}^{\prime \prime}\left(b-b_{s}\right)^{3}\right) .
$$

The quadratic part is also the classical expression of the potential energy for internal gravity waves, derived for instance in Gill (1982).

Decomposing the spatial integral of (4.5) into a sum of integrals over each macrocell of the discrete model depicted in figure 1 and taking the limit of an infinite number of macrocells, the potential energy can be expressed in terms of the local variance of buoyancy fluctuations:

$$
E_{p}=\frac{V}{4 H} \int_{-H}^{+H} \mathrm{~d} z \frac{\overline{b^{2}}-\bar{b}^{2}}{b_{s}^{\prime}}+O\left(Z_{s}^{\prime \prime} \overline{\left(b-b_{s}\right)^{3}}\right) .
$$

The variance of buoyancy fluctuations $\overline{b^{2}}-\bar{b}^{2}$ is related to the local kinetic energy $e_{c}$ and the local buoyancy gradient $\mathrm{d} \bar{b} / \mathrm{d} z$ though (2.21). Inserting this equation into (4.6), using $\mathrm{d} \bar{b} / \mathrm{d} z=b_{s}^{\prime}$ (which is valid for sufficiently large Richardson numbers) and $e_{c}=E_{c} / V$ :

$$
E_{p}=\frac{E_{c}}{3},
$$

which shows equipartition of the energy between the available potential energy and the three degrees of freedom of the kinetic energy. A direct consequence of energy equipartition is thus

$$
\eta=\frac{1}{4} .
$$

This result is a direct consequence of the quadratic form of the energy obtained in (4.6), which relies on the assumptions (i) that $b_{s}^{\prime}$ is strictly positive and bounded, (ii) that $Z_{s}^{\prime \prime}$ is bounded, (iii) that $b$ remains sufficiently close to $b_{s}$.

Importantly, the hypotheses (i) and (ii) are not satisfied when the background buoyancy profile contains homogeneous layers of fluids, as for instance in the case depicted in figure $3(a)$. In order to evaluate when the assumption (iii) is valid, one can estimate the typical value of $b-b_{s}$ at a given point as the root mean square of local buoyancy fluctuations $\left(\overline{b^{2}}-\bar{b}^{2}\right)^{1 / 2}$. Using (2.21), $\partial_{z} \bar{b}=\partial_{z} b_{s}$ and $R i \sim H^{2} b_{s}^{\prime} / e_{c}$ yields then $\left(b-b_{s}\right) \sim R i^{-1 / 2}$.

We conclude that $\eta=0.25$ is expected in the limit of large Richardson number, when the background buoyancy profile is strictly increasing with height.

\subsection{Comparison with previous studies of the efficiency of mixing}

Despite the large number of numerical and experimental studies devoted to the understanding of mixing efficiency, there are only few theoretical results yielding predictions for the variations of mixing efficiency with the Richardson number. In the 
context of shear-stratified turbulence, dimensional analysis was used by Townsend (1958) to model the variation of mixing efficiency with the gradient Richardson number and upper bounds for the mixing efficiency have been derived rigorously by Caulfield \& Kerswell (2001).

A mixing efficiency $\eta=0.25$ was obtained by a phenomenological model due to McEwan (1983b), based on purely kinematic arguments. Those predictions were found to be consistent with experimental observations of mixing efficiency following an internal wave-breaking event (McEwan 1983a). The argument is the following: take a continuously stratified fluid at rest, and exchange two particle fluids $a$ and $b$ of volume $\delta V$ with buoyancy difference $\Delta b=b_{b}-b_{a}$ and height difference $\Delta z=z_{b}-z_{a}$, with $\delta V / V \ll \Delta z / H$. Then consider the small displacement limit $\Delta z \rightarrow 0$, which, as explained in previous sections, corresponds to a weak energy limit, or equivalently to a large Richardson number limit, for which $\Delta b=\Delta z \mathrm{~d} \bar{b} / \mathrm{d} z$. Given that the injected energy is under the form of available potential energy only, the initial kinetic energy is zero, with $E_{i n j}=b_{s}^{\prime}(\Delta z)^{2} \delta V$. McEwan (1983b) then argued that the two displaced fluid particles will be stirred and mix together until homogenisation of their buoyancy, and that the two fluid particles carrying buoyancy $\left(b_{a}+b_{b}\right) / 2$ will 'sediment' to their rest position $z=\left(z_{a}+z_{b}\right) / 2$. The available potential energy of the final state is then $E_{p}=b_{s}^{\prime}(\Delta z / 2)^{2} \delta V$, which corresponds to mixing efficiency $\eta=0.25$.

Strikingly, several numerical studies have also reported convergence of mixing efficiency towards $\eta=0.25$ at large Richardson numbers; see e.g. Maffioli, Brethouwer \& Lindborg (2016), Venayagamoorthy \& Koseff (2016) and references therein. (Maffioli et al. (2016) report a mixing coefficient $\Gamma=\eta /(1-\eta)=0.33$ in the limit of small Froude numbers, which corresponds to $\eta=0.25$ in the limit of large Richardson numbers.) It is remarkable that the statistical mechanics theory in the large Richardson number limit also yields $\eta=0.25$, provided that the initial buoyancy is strictly monotonic. We stress that the only assumption underlying the equilibrium theory is that the system evenly explores the phase space: there is neither dynamics nor kinematics involved in the derivation of this result. By contrast, the approach of McEwan (1983b) relies on the choice of a peculiar kinematic model.

McEwan (1983b) also discussed the case of two homogeneous layers separated by a linear pycnocline of thickness $\delta$. He found that mixing efficiency vanishes when considering first the limit $\delta \rightarrow 0$ and second the limit of large Richardson numbers $R i \rightarrow+\infty$. This is again fully consistent with the statistical mechanics predictions for the mixing efficiency in the two-level case depicted in figure 3(a). Indeed, it corresponds to the case of a background buoyancy profile with an infinity sharp interface. The mixing efficiency vanishes in the limit of infinite Richardson numbers because kinetic energy is spread equally over the whole domain at equilibrium, while buoyancy mixing is confined to a thin layer surrounding the buoyancy interface, with a thickness that decrease with the Richardson number, as explained in $\S 3.1$.

We stress that the statistical theory makes possible predictions for global, cumulative mixing efficiency in decaying turbulence whatever the Richardson number, and whatever the background buoyancy profile. In particular, it predicts a bell shape for $\eta(R i)$ in the two-layer case, with a maximum $\eta=0.15$, and a monotonic increase of $\eta(R i)$ in the linear case from $\eta=0$ to $\eta=0.25$, as shown in figure 3 . The bell shape for $\eta(R i)$ has been reported in decaying experiments performed by dropping a grid in a two-layer stratified fluid (Linden 1980), but how to estimate the amount of energy injected into the system in experiments continue to be debated, see e.g. Huq \& Britter (1995). The monotonic increase of cumulative mixing efficiency in the case of a linear background buoyancy profile seems a robust result in laboratory and 
numerical experiments, see e.g. Stretch et al. (2010). However, the equilibrium theory does not account for layering which is often observed in the strongly stratified regime $R i \gg 1$ (Rehmann \& Koseff 2004). In any case, the statistical mechanics prediction that mixing efficiency depends strongly on the global shape of the background buoyancy profile, and not only on the local buoyancy gradient is consistent with observations by Holford \& Linden (1999).

There is however one result that does not depend on the shape of the buoyancy profile: according to the equilibrium theory, the mixing efficiency should increase linearly with the Richardson numbers in the limit of weak Richardson numbers. This scaling law can be simply understood as a consequence of the fact that buoyancy behaves as a passive tracer in this limit (Holford \& Linden 1999). This linear scaling has been also reported by Maffioli et al. (2016) in forced-dissipative numerical experiments, who also provide complementary arguments based on cascade phenomenology.

The observations mentioned in the above paragraphs provide support for the statistical mechanics predictions. Other observations however point to limitations of the theory. For instance, the predicted value of mixing efficiency in decaying turbulence depends on the total energy injected into the system, but not on how the energy is injected. Yet different values of mixing efficiency have been reported in laboratory and numerical experiments performed with different energy injection mechanism. A value $\eta \approx 0.2$ was reported in decaying shear-stratified fluids with a Richardson number of order one (Peltier \& Caulfield 2003). This value is somewhat larger than the cumulative mixing efficiency $\eta=0.11$ observed in lock-exchange experiments (Prastowo et al. 2008; Ilicak 2014), and smaller than the cumulative mixing efficiency $\eta \approx 0.5$ reported in the framework of Rayleigh-Taylor experiments (Dalziel et al. 2008; Davis Wykes \& Dalziel 2014). Importantly, these different values for mixing efficiency do not depend only on the Richardson, Reynolds and Péclet numbers. This suggests that the mechanism of injection plays an important role.

A heuristic way to discuss more precisely the role of the injection mechanism in relation with the ergodicity hypothesis is to consider the parameter $L_{t} / H$, i.e. the ratio of the energy injection length scale to the domain scale. In the context of two-dimensional turbulence, this parameter has been proven useful to discuss the relevance of the ergodic hypothesis underlying statistical mechanics theory (Pomeau 1994; Tabeling 2002; Venaille, Dauxois \& Ruffo 2015). Denoting $T_{\text {tran }}$ the typical time scale to move a fluid particle from the top to the bottom of the tank through turbulent transport, and calling $T_{\text {diss }}=L_{t} / U$ the typical time scale for the dissipation rates for local buoyancy fluctuations through direct turbulent cascade, the system can explores the phase space only if $T_{\text {trans }}<T_{\text {diss }}$. Modelling turbulent transport as an effective eddy viscosity or eddy diffusivity $U L_{t}$ yields $T_{\text {trans }}=H^{2} /\left(U L_{t}\right)$ and then the necessary condition $H<L_{t}$ for ergodicity. Given that $L_{t}$ cannot be larger than the domain height, we see that the condition for sufficient mixing in phase space will only be marginally satisfied when $H=L_{t}$, and will not be satisfied when $L_{t} \ll H$.

Finally, the equilibrium theory applies in principle to flow systems in the limit of infinitely large Reynolds and Péclet numbers. Even if it is natural to expect that the dissipation rate of buoyancy and kinetic energy become independent from the value of molecular viscosity and diffusivity when they are sufficiently weak, numerical and laboratory experiments are often performed in intermediate regimes for which those parameters may influence the mixing efficiency, see e.g. Shih et al. (2005), Bouffard \& Boegman (2013), Lozovatsky \& Fernando (2013), Salehipour \& Peltier (2015). 


\section{Conclusion}

We have addressed the problem of mixing efficiency from the point of view of equilibrium statistical mechanics. The theory predicts that the unforced, inviscid, adiabatic dynamics is attracted towards a state characterised by small-scale velocity fluctuations carrying kinetic energy, and by a smooth, monotonic buoyancy profile upon which are small-scale buoyancy fluctuations. Although the whole dynamics is adiabatic, the buoyancy field is irreversibly mixed at a coarse-grained level, no matter how small the coarse-grained scale. In addition, the coarse-grained fields predicted by the theory are stationary and characterised by a stable buoyancy profile. The theory also predicts velocity fluctuations to be Gaussian, isotropic, homogeneous in space, and that the buoyancy fluctuations are homogeneous on horizontal planes.

The input of the theory is the total energy injected initially into the system, and the global distribution of buoyancy levels, or equivalently the background buoyancy profile. The output of the theory is the probability to measure a given buoyancy level at each height. We provide explicit computations of the equilibria in limiting cases, and implement an algorithm based on a maximum entropy production which determines the equilibrium state for any background buoyancy profile. This allows us to compute a cumulative mixing efficiency defined as the ratio of the potential energy gained by the system to the total energy injected into the system. Importantly, the potential energy effectively gained by the system is the potential energy of the coarse-grained buoyancy profile at equilibrium minus the potential energy of the background buoyancy profile. The background potential energy remains constant for the adiabatic dynamics, but the irreversible convergence of the system towards the equilibrium state implies an irreversible increase of potential energy for the system. Several results on the cumulative mixing efficiency are obtained within this framework.

(1) The cumulative mixing efficiency increases in proportion to the Richardson number in the limit of small Richardson number, whatever the background buoyancy profile.

(2) The cumulative mixing efficiency tends to 0.25 in the limit of infinite Richardson numbers, provided that the background buoyancy profile is strictly decreasing with height (no homogeneous layer).

(3) The variations of the cumulative mixing efficiency with the Richardson number depends strongly on the background buoyancy profile, and can be non-monotonic. In the particular case of a fluid with two homogeneous layers of different buoyancy, the theory predicts a bell shape for the cumulative mixing efficiency as a function of the global Richardson number.

The application of equilibrium statistical mechanics to mixing in stratified fluids however relies on two key hypotheses.

(i) The theory applies to inviscid, adiabatic Boussinesq fluids. It is expected to describe mixing only in the limit of high Reynolds and Péclet numbers.

(ii) Equilibrium statistical mechanics relies on the counting of the available microscopic states, and its predictive power depends on the capability of the system to actually explore those available states. Such an ergodic behaviour is favoured by stirring at the system scale $H$. By contrast turbulence forced at small scale $L_{t}$ with $L_{t} / H<1$ is expected to produce local mixing before large-scale stirring, leading to discrepancies of the statistical mechanics predictions. 
Note finally, the theory does not predict how the system converges towards equilibrium. It does neither predict a turbulent diffusivity nor a turbulent viscosity during the relaxation process, but only the final outcome of turbulent stirring. And it does not account for energy fluxes in forced-dissipative configurations.

Equilibrium statistical mechanics therefore describes an ideal state of inviscid stirring which is not fully reached in most cases. Turbulent stirring could be however modelled locally as a trend to approach this equilibrium. This can be done by giving a dynamical meaning to the relaxation equations used in this paper as an algorithm to compute the equilibrium state. Indeed, those equations contain a classical term modelling turbulent transport as an effective diffusion, with an additional drift term describing restratification. We believe that this approach will be fruitful to model relaminarisation after a mixing event, see e.g. Venaille \& Sommeria (2010). This would provide a complementary point of view to other statistical or stochastic approaches that have long been used in the context of combustion (Pope 1985), and adapted to the case of turbulent mixing in stratified fluids (Kerstein 1999). We hope the present paper will motivate further studies in those directions.

\section{Acknowledgements}

We warmly thank T. Dauxois, C. Herbert, P. Odier, A. Renaud and C. Staquet for useful discussions. Part of this work has been funded by ANR-13-JS09-0004-01 (STRATIMIX).

\section{Appendix A. Liouville theorem}

We show in this appendix that the quadruplet of fields $(\boldsymbol{u}, b)$ satisfy a Liouville theorem, i.e. that trajectories of the system are non-divergent in a phase space described by this quadruplet of fields. The fact that Fourier components of the velocity field in each direction satisfy a detailed Liouville theorem is a classical result for three-dimensional Euler dynamics (Lee 1952). Generalisation of this results to the inviscid, adiabatic Boussinesq system is straightforward, but is reproduced here for completeness. Let us for that purpose decompose both the velocity field and the buoyancy field on Fourier modes:

$$
\boldsymbol{u}=\sum_{\boldsymbol{k}} \hat{\boldsymbol{u}}_{\boldsymbol{k}}(t) \mathrm{e}^{\mathrm{i} k \cdot \boldsymbol{x}}, \quad b=\sum_{\boldsymbol{k}} \hat{b}_{\boldsymbol{k}}(t) \mathrm{e}^{\mathrm{i} \boldsymbol{k} \cdot \boldsymbol{x}} .
$$

Writing $\boldsymbol{u}=\left(u_{1}, u_{2}, u_{3}\right)$ and projecting the equations of motion (2.1)-(2.3) on a mode $\boldsymbol{k}$ yields

$$
\begin{gathered}
\dot{\hat{b}}_{\boldsymbol{k}}=-\mathrm{i} \sum_{\boldsymbol{p}+q=k}\left(\hat{\boldsymbol{u}}_{\boldsymbol{p}} \cdot \boldsymbol{q}\right) \hat{b}_{\boldsymbol{q}}, \\
\dot{\hat{u}}_{i k}=\sum_{j, l}\left[\left(\delta_{i 3}-\frac{k_{3} k_{i}}{k^{2}}\right) \hat{b}_{\boldsymbol{k}}+\left(\frac{k_{i} k_{j}}{k^{2}}-\delta_{i j}\right) \sum_{\boldsymbol{p}+q=k} q_{l} \hat{u}_{l p} \hat{u}_{j q}\right] .
\end{gathered}
$$

The pressure term has been eliminated from the momentum equation by using the non-divergence condition. Deriving (A 2) by $b_{k}$ and (A 3) by $u_{i k}$ allows us to show the existence of a detailed Liouville theorem for the Fourier components of the buoyancy field $b$, and for the Fourier components of the velocity field in each direction:

$$
\forall \boldsymbol{k}, \quad \frac{\partial \dot{b}_{k}}{\partial \hat{b}_{k}}+\frac{\partial \dot{\hat{b}}_{-k}}{\partial \hat{b}_{-k}}=0, \quad \text { and } \quad \forall i, \quad \boldsymbol{k} \quad \frac{\partial \dot{\hat{u}}_{i k}}{\partial \hat{u}_{i k}}+\frac{\partial \dot{\hat{u}}_{i-k}}{\partial \hat{u}_{i-k}}=0 .
$$


Using $\left(u_{1}, u_{2}, u_{3}\right)=(u, v, w)$, we conclude that the quadruplet of fields $(u, v, w, b)$ satisfies a Liouville theorem:

$$
\sum_{k}\left[\frac{\partial \dot{\hat{b}}_{k}}{\partial \hat{b}_{k}}+\frac{\partial \dot{\hat{u}}_{k}}{\partial \hat{u}_{k}}+\frac{\partial \dot{\hat{v}}_{k}}{\partial \hat{v}_{k}}+\frac{\partial \dot{\hat{w}}_{k}}{\partial \hat{w}_{k}}\right]=0 .
$$

This Liouville theorem expresses the conservation of volume in the space of spectral amplitudes. However the discrete approximation of the fields that we propose in this paper relies on a uniform microscopic grid in physical space, and one needs to show that the Liouville property is not broken by this discrete approximation. We note for that purpose (i) that the Liouville property in (A 5) remains valid if the sum is truncated at wavenumbers $k_{i} \leqslant N / 2$ for $1 \leqslant i \leqslant 3$, whatever the value of $N$, and (ii) that for a given truncation of the fields in Fourier space, the spectral amplitudes are related to the values of the fields on a collocation grid uniform in physical space, through a linear transformation that does not depend on the fields. The Jacobian of the transformation is therefore an unimportant constant, as noted in Miller (1990). We conclude that a Liouville theorem holds for the finite-dimensional approximation of the buoyancy and velocity fields on a uniform grid.

\section{Appendix B. From Boltzmann entropy to macrostate entropy}

The aim of this appendix is to count the number of microscopic configurations $\boldsymbol{u}(\boldsymbol{x}), b(\boldsymbol{x})$ associated with a given macroscopic state $\rho(\boldsymbol{x}, \sigma, \boldsymbol{v})$. In order to simplify the presentation, we show first how to count the number of microscopic configurations $b(\boldsymbol{x})$ associated with a given macroscopic state $\rho(\boldsymbol{x}, \sigma)$. The first step is to introduce a discrete approximation of the fields. The second step is a classical counting arguments within each macrocell of the discrete model. The third step is to consider the limit of an infinite number of grid point within each macrocell, which corresponds to the continuous limit for the microscopic configurations. The last step is to consider the limit of an infinite number of macrocells, which corresponds to the continuous limit for the macroscopic states, or equivalently to the limit of a vanishing coarse-graining length scale.

We assume that the domain $\mathcal{V}_{x}$ is divided into a uniform grid containing $N$ cubic macrocells indexed by $1 \leqslant I \leqslant N$, and that each macrocell is divided into another uniform grid containing $M$ sites, where each site contains one and only one fluid particle indexed by $1 \leqslant i \leqslant M$, see figure 1 . We also assume that the buoyancy $b_{I, i}$ at site $(I, i)$ can only take on a discrete number of values (say $K$ ), with $b_{I, i} \in\left\{\sigma_{1}, \ldots, \sigma_{K}\right\}$, and that each of the resulting microstates is equiprobable. We note that with this procedure, we count the fields that will not be differentiable when taking the continuous limit, and we will see that the equilibrium state is actually characterised by such states containing fluctuations of buoyancy at scale smaller than a coarse-grained scale, no matter how small the coarse-grained scale is. For a given discretised buoyancy field, we call $M_{I, k}$ the number of fluid particles carrying the buoyancy level $\sigma_{k}$ within the macrocell $I$, and $M_{I, k} / M$ is therefore the frequency of occurrence of the level $\sigma_{k}$ at site $I$ for one realisation of the discretised field. The system is described at a macroscopic level by the probability $p_{I, k}$ of measuring the buoyancy level $\sigma_{k}$ at site $I$.

Our aim is to count number of microscopic configurations associated with a prescribed field $p_{I, k}$. We use for that purpose the equivalence between probability and frequency in the large $M$ limit:

$$
p_{I, k}=\lim _{M \rightarrow+\infty} \frac{M_{I, k}}{M} .
$$


In the large $M$ limit, the number of microscopic discretised buoyancy fields $\left\{b_{I, i}\right\}_{1 \leqslant I \leqslant N, 1 \leqslant i \leqslant M}$ associated with the macroscopic field $\left\{p_{I, k}\right\}_{1 \leqslant I \leqslant N, 1 \leqslant k \leqslant K}$ is

$$
\Omega=\prod_{I=1}^{N}\left(\frac{M !}{\prod_{k=1}^{K}\left(M p_{I, k}\right) !}\right) .
$$

The Boltzmann entropy is defined as

$$
S_{B}=k_{B} \log \Omega,
$$

where $k_{B}$ is a constant. In the large $M$ limit, the Stirling formula $(\log M !=M \log M)$ leads at lowest order to

$$
S_{B}=-k_{B} M \sum_{I=1}^{N} \sum_{k=1}^{K} p_{I, k} \log p_{I, k},
$$

where we have kept only the dominant term, and removed an unimportant constant depending on the grid size $M$.

It is important to note that for a given macrostate $\left\{p_{I, k}\right\}_{1 \leqslant I \leqslant N, 1 \leqslant k \leqslant K}$, the number of possible microscopic configurations $\Omega$ diverges exponentially with $M$, which a coefficient given by $-\sum_{I=1}^{N} \sum_{k=1}^{K} p_{I, k} \log p_{I, k}$. This means that among a set of different macrostates, there will be an overwhelming number of microstates associated with the one that maximises the coefficient $-\sum_{I=1}^{N} \sum_{k=1}^{K} p_{I, k} \log p_{I, k}$. In other words, a single microscopic configuration picked up at random has a very large probability of being close to the macroscopic equilibrium state. A practical consequence of this concentration property is that no particular average procedure is required to observe the actual macroscopic equilibrium state.

In the limit $N \rightarrow+\infty$, the sum over $I$ in (B 4), can be replaced by an integral over the spatial coordinate $\boldsymbol{x}$ if the discretised probability field $\left\{p_{I, k}\right\}_{1 \leqslant I \leqslant N, 1 \leqslant k \leqslant K}$ is also replaced by its continuous counterpart $\left\{p_{K}(\boldsymbol{x})\right\}_{1 \leqslant k \leqslant K}$ :

$$
S_{B}=-k_{B} \frac{M N}{V} \int_{\mathcal{V}_{\boldsymbol{x}}} \mathrm{d} \boldsymbol{x} \sum_{k=1}^{K} p_{k}(\boldsymbol{x}) \log p_{k}(\boldsymbol{x}) .
$$

Note that the quantity $p_{k}(\boldsymbol{x})$ is normalised at each point $\boldsymbol{x}$, with $\sum_{k=1}^{K} p_{k}(\boldsymbol{x})=1$. It describes the local fluctuations of the (continuous) microscopic field $b$ in the vicinity of point $\boldsymbol{x}$, and it is called a Young measure in mathematics.

A generalisation to the case of a continuum of buoyancy levels $\sigma \in \mathcal{V}_{\sigma}=\left[\sigma_{\min } \sigma_{\max }\right]$ with probability density function $\rho(\sigma, \boldsymbol{x})$ is less straightforward and requires the use of Sanov's theorem, see e.g. Touchette (2009). However, the result is easily inferred from (B 5) by decomposing the interval $\left[\sigma_{\min } \sigma_{\max }\right]$ into $K$ levels $\sigma_{k}$ equally spaced with interval $\Delta \sigma$, and by considering $\rho\left(\boldsymbol{x}, \sigma_{k}\right)=p_{k}(\boldsymbol{x}) / \Delta \sigma$. Taking the limit $K \rightarrow+\infty$ yields

$$
S_{B}=-k_{B} \frac{M N}{V} \int_{\mathcal{V}_{x}} \mathrm{~d} \boldsymbol{x} \int_{\mathcal{V}_{\sigma}} \mathrm{d} \sigma \rho(\boldsymbol{x}, \sigma) \log \rho(\boldsymbol{x}, \sigma),
$$

up to an unimportant term depending on $K$. The quantity $\rho(\boldsymbol{x}, \sigma)$ is now the probability density function of measuring the buoyancy level $b=\sigma$ at height $z$, with the normalisation constraint $\int_{\mathcal{V}_{\sigma}} \mathrm{d} \sigma \rho(\boldsymbol{x}, \sigma)=1$. 
We are now ready to generalise this result to the case where a fluid particle at point $\boldsymbol{x}$ is carrying not only a buoyancy level $b(\boldsymbol{x})=\sigma$ with $\sigma \in \mathcal{V}_{\sigma}$, but also a velocity (vector) level $\boldsymbol{u}(\boldsymbol{x})=\boldsymbol{v}$ with $\boldsymbol{v} \in \mathcal{V}_{v}=\left[-v_{\max }, v_{\max }\right]^{3}$. The same steps leading to (B 6) can be applied to that case, which yields

$$
S_{B}=-\frac{k_{B} M N}{V} \int_{\mathcal{V}_{x}} \mathrm{~d} \boldsymbol{x} \int_{\mathcal{V}_{\sigma}} \mathrm{d} \sigma \int_{\mathcal{V}_{v}} \mathrm{~d} \boldsymbol{v} \rho(\boldsymbol{x}, \sigma, \boldsymbol{v}) \log \rho(\boldsymbol{x}, \sigma, \boldsymbol{v}),
$$

where $\rho(\boldsymbol{x}, \sigma, \boldsymbol{v})$ is the probability density function for the buoyancy and velocity at point $\boldsymbol{x}$. Note that we have introduced a cutoff denoted $v_{\max }$ for the maximum possible velocity. Anticipating that velocity fluctuations are bounded due to the energy constraint, we expect that the results will not depend on $v_{\max }$ if it is chosen much larger than the root mean square velocity of the equilibrium state, and we will consider in the remaining of this paper $v_{\max }=+\infty$.

Finally, choosing $k_{B}=V /(N M)$ in (B 7), we recover $S_{B}=\mathcal{S}[\rho]$, where $\mathcal{S}[\rho]$ is the macrostate entropy defined in (2.13).

\section{Appendix C. Relaxation equations from a maximum entropy production principle}

The aim of this appendix is to provide an algorithm that makes possible numerical computations of the equilibrium states for arbitrary energy $E$ and global distribution of buoyancy $G(\sigma)$. We consider for that purpose the ansatz (3.6) for the local distribution of velocity and buoyancy levels, and we propose in the following a dynamical system describing the temporal evolution of the quantities $\rho_{b}(\boldsymbol{x}, \sigma, t), e_{c}(t)$ in such a way that the total energy and the global distribution of buoyancy levels are conserved, just as in the original Boussinesq system, and in such a way that the entropy production is maximum at each time. This maximum entropy production principle ensures convergence towards an entropy maximum for a given set of constraints $E$, $G(\sigma)$. Since the effective temperature (i.e. the Lagrange parameter associated with the energy) is positive, the entropy maximum is unique for a given set of constraints, and the dynamical system will therefore relax towards the equilibrium state. We stress that considering the temporal evolution of this dynamical system is a trick to find the equilibrium state. The actual flow dynamics may follow a different path towards equilibrium than the one maximizing the entropy production.

Since the dynamical system is fully described by $\rho_{b}(\boldsymbol{x}, \sigma, t)$ and $e_{c}(t)$, it will be useful in the following to express the conservation of the global buoyancy distribution and of the total energy in terms of those parameters. Inserting (3.6) in (2.12) and (2.11) yields

$$
\begin{gathered}
\mathcal{G}\left[\rho_{b}\right]=\frac{V}{2 H} \int_{-H}^{H} \mathrm{~d} z \rho_{b}, \\
\mathcal{E}\left[\rho_{b}\right]\left(e_{c}\right)=V e_{c}+\frac{V}{2 H} \int_{-H}^{H} \mathrm{~d} z \int_{\mathcal{V}_{\sigma}} \mathrm{d} \sigma \rho_{b} \sigma z .
\end{gathered}
$$

If the initial condition $\rho_{b}(z, \sigma, 0)$ and the initial kinetic energy $e_{c}(0)$ are known, then the global distribution of buoyancy levels and total energy can be computed using (C 1) and (C2), respectively.

Assuming that there is no source nor sink of density, recalling that the flow is nondivergent, and anticipating that there is no mean flow, the temporal evolution of the pdf $\rho_{b}$ satisfies the general conservation law

$$
\partial_{t} \rho_{b}+\partial_{z} J_{b}=0
$$


where we have introduced the turbulent flux of probability $J_{b}(z, \sigma, t)$ directed along $z$, with $J_{b}=0$ at the upper and the lower boundary $z= \pm H$.

The temporal evolution of the system requires a model for the flux $J_{b}$ and the kinetic energy production $\dot{e}_{c}=\mathrm{d} e_{c} / \mathrm{d} t$. The maximum entropy production principle amounts to finding the flux $J_{b}$ and the kinetic energy production $\dot{e}_{c}$ that maximise the entropy production while satisfying the constraints of the problem.

Let us first compute the entropy and energy production. Injecting the ansatz (3.6) in (2.13), the macrostate entropy can be expressed as

$$
\mathcal{S}=-\frac{V}{2 H} \int_{-H}^{+H} \mathrm{~d} z \int_{\mathcal{V}_{\sigma}} \mathrm{d} \sigma \rho_{b} \log \rho_{b}-\frac{3}{2} V \log \frac{3}{2 e_{c}} .
$$

Taking the time derivative of (C4) and using (C 3), the entropy production can be expressed as

$$
\dot{\mathcal{S}}=\frac{V}{2 H} \int_{-H}^{+H} \mathrm{~d} z \int_{\mathcal{V}_{\sigma}} \mathrm{d} \sigma J_{b} \partial_{z}\left(\log \rho_{b}\right)-\frac{3}{2} V \frac{\dot{e}_{c}}{e_{c}} .
$$

The constraints of the problem are given by

(i) the conservation of the local normalisation (2.7), implying

$$
\forall z \in[-H H], \quad \int_{\mathcal{V}_{\sigma}} \mathrm{d} \sigma J_{b}=0,
$$

which ensures the local normalization $\int_{\mathcal{V}_{\sigma}} \mathrm{d} \sigma \rho_{b}=1$,

(ii) the energy conservation, which can be expressed as $\dot{\mathcal{E}}=0$. Taking the temporal derivative of (C2) yields

$$
\dot{\mathcal{E}}=V \dot{e}_{c}+\frac{V}{2 H} \int_{-H}^{+H} \mathrm{~d} z \int_{\mathcal{V}_{\sigma}} \mathrm{d} \sigma \sigma J_{b} .
$$

(iii) Finally, the fluxes of probability must be finite to be dynamically relevant. Indeed, an infinite flux would correspond to an instantaneous rearrangement of the buoyancy field. We impose therefore a bound for the norm of the probability flux $J_{b}$, expressed as:

$$
\forall z \in[-H H], \quad \int_{\mathcal{V}_{\sigma}} \mathrm{d} \sigma \frac{J_{b}^{2}}{2 \rho_{b}} \leqslant C(z) .
$$

The quantity $J_{b} / \rho_{b}$ can be interpreted as a diffusion velocity for the probability density field, and the constraint in (C 8) ensures that this velocity remains finite everywhere and for each buoyancy level during the relaxation process.

The variational problem of the maximum entropy production principle is treated by introducing Lagrange multipliers $\zeta(z), \beta_{t}$ and $-/ D(z)$ associated with the constraints in (C 6), (C 7) and (C 8), respectively. Note that following the Karush-Kuhn-Tucker conditions, an inequality such as (C8) can be treated as an equality constraint when computing the first-order variations in an optimisation problem (Sundaram 1996). The condition

$$
\delta \dot{\mathcal{S}}-\beta_{t} \delta \dot{\mathcal{E}}+\int_{-H}^{+H} \mathrm{~d} z \int_{\mathcal{V}_{\sigma}} \mathrm{d} \sigma \zeta(z) \delta J_{b}-\int_{-H}^{+H} \mathrm{~d} z \int_{\mathcal{V}_{\sigma}} \mathrm{d} \sigma \frac{1}{D} \frac{J_{b}}{\rho_{b}} \delta J_{b}=0
$$


must be satisfied for each $\delta \dot{e}_{c}$ and $\delta J_{b}$. Using

$$
\left.\begin{array}{c}
\delta \dot{\mathcal{S}}=\frac{V}{2 H} \int_{-H}^{+H} \mathrm{~d} z \int_{\mathcal{V}_{\sigma}} \mathrm{d} \sigma \partial_{z}\left(\log \rho_{b}\right) \delta J_{b}-\frac{3 V}{2 e_{c}} \delta \dot{e}_{c}, \\
\delta \dot{\mathcal{E}}=V \delta \dot{e}_{c}+\frac{V}{2 H} \int_{-H}^{+H} \mathrm{~d} z \int_{\mathcal{V}_{\sigma}} \mathrm{d} \sigma \sigma \delta J_{b},
\end{array}\right\}
$$

equation (C9) yields

$$
\beta_{t}=3 /\left(2 e_{c}\right), \quad J_{b}=-D\left(\partial_{z} \rho_{b}-\beta_{t}(\sigma-\bar{b}) \rho_{b}\right),
$$

where $\zeta(z)$ has been determined by using the constraint in (C6). In addition, the coefficient $D$ must be positive for the entropy production to be positive. As far as the equilibrium state is concerned, the value of $D$ in not important. Indeed, the flux $J_{b}$ vanishes when equilibrium is reached, which ensures that the equilibrium state does not depend on $D$.

\section{REFERENCES}

BARTEllo, P. 1995 Geostrophic adjustment and inverse cascades in rotating stratified turbulence. J. Atmos. Sci. 52 (24), 4410-4428.

Boucher, C., Ellis, R. S. \& Turkington, B. 2000 Derivation of maximum entropy principles in two-dimensional turbulence via large deviations. J. Stat. Phys. 98 (5-6), 1235-1278.

Bouchet, F. \& Corvellec, M. 2010 Invariant measures of the 2d Euler and Vlasov equations. J. Stat. Mech. 2010 (08), P08021.

Bouchet, F. \& Simonnet, E. 2009 Random changes of flow topology in two-dimensional and geophysical turbulence. Phys. Rev. Lett. 102 (9), 094504.

Bouchet, F. \& Sommeria, J. 2002 Emergence of intense jets and jupiter's great red spot as maximum-entropy structures. J. Fluid Mech. 464, 165-207.

Bouchet, F. \& Venaille, A. 2012 Statistical mechanics of two-dimensional and geophysical flows. Phys. Rep. 515 (5), 227-295.

Bouffard, D. \& Boegman, L. 2013 A diapycnal diffusivity model for stratified environmental flows. Dyn. Atmos. Oceans 61, 14-34.

Caulfield, C. P. \& Kerswell, R. R. 2001 Maximal mixing rate in turbulent stably stratified couette flow. Phys. Fluids 13 (4), 894-900.

Chavanis, P.-H. 2002 Statistical mechanics of two-dimensional vortices and stellar systems. In Dynamics and Thermodynamics of Systems with Long-range Interactions, pp. 208-289. Springer.

CoRrsin, S. 1951 On the spectrum of isotropic temperature fluctuations in an isotropic turbulence. J. Appl. Phys. 22 (4), 469-473.

Dalziel, S. B., Patterson, M. D., Caulfield, C. P. \& Coomaraswamy, I. A. 2008 Mixing efficiency in high-aspect-ratio Rayleigh-Taylor experiments. Phys. Fluids 20 (6), 065106.

Davis Wykes, M. S., Hughes, G. O.\& Dalziel, S. B. 2015 On the meaning of mixing efficiency for buoyancy-driven mixing in stratified turbulent flows. J. Fluid Mech. 781, 261-275.

Davis WyKes, M. S. D. \& DAlziel, S. B. 2014 Efficient mixing in stratified flows: experimental study of a Rayleigh-Taylor unstable interface within an otherwise stable stratification. J. Fluid Mech. 756, 1027-1057.

Eyink, G. L. \& SREenivasan, K. R. 2006 Onsager and the theory of hydrodynamic turbulence. Rev. Mod. Phys. 78 (1), 87.

Falkovich, G., Gawesdzki, K. \& Vergassola, M. 2001 Particles and fields in fluid turbulence. Rev. Mod. Phys. 73 (4), 913.

Fernando, H. J. S. 1991 Turbulent mixing in stratified fluids. Annu. Rev. Fluid Mech. 23, 455-493.

Gill, A. E. 1982 Atmosphere-ocean Dynamics, vol. 30. Academic. 
Herbert, C., Pouquet, A. \& Marino, R. 2014 Restricted equilibrium and the energy cascade in rotating and stratified flows. J. Fluid Mech. 758, 374-406.

Holford, J. M. \& Linden, P. F. 1999 Turbulent mixing in a stratified fluid. Dyn. Atmos. Oceans 30 (2), 173-198.

Hopfinger, E. J. 1987 Turbulence in stratified fluids: a review. J. Geophys. Res. 92 (C5), 5287-5303.

HUQ, P. \& BRITTER, R. E. 1995 Turbulence evolution and mixing in a two-layer stably stratified fluid. J. Fluid Mech. 285, 41-68.

ILICAK, M. 2014 Energetics and mixing efficiency of lock-exchange flow. Ocean Model. 83, 1-10.

IVey, G. N., Winters, K. B. \& KosefF, J. R. 2008 Density stratification, turbulence, but how much mixing? Annu. Rev. Fluid Mech. 40 (1), 169.

Kerstein, A. R. 1999 One-dimensional turbulence: model formulation and application to homogeneous turbulence, shear flows, and buoyant stratified flows. J. Fluid Mech. 392, 277-334.

Kraichnan, R. H. 1967 Inertial ranges in two-dimensional turbulence. Phys. Fluids 10, 1417-1423.

Large, W. G., MCWilliams, J. C. \& Doney, S. C. 1994 Oceanic vertical mixing: a review and a model with a nonlocal boundary layer parameterization. Rev. Geophys. 32 (4), 363-404.

LEE, T. D. 1952 On some statistical properties of hydrodynamical and magnetohydrodynamical fields. Q. Appl. Maths 10 (1), 69-74.

LINDBORG, E. 2005 The effect of rotation on the mesoscale energy cascade in the free atmosphere. Geophys. Res. Lett. 32 (1).

Lindborg, E. 2006 The energy cascade in a strongly stratified fluid. J. Fluid Mech. 550, 207-242.

Linden, P. F. 1979 Mixing in stratified fluids. Geophys. Astrophys. Fluid Dyn. 13 (1), 3-23.

Linden, P. F. 1980 Mixing across a density interface produced by grid turbulence. J. Fluid Mech. 100 (04), 691-703.

Lozovatsky, I. D. \& Fernando, H. J. S. 2013 Mixing efficiency in natural flows. Phil. Trans. R. Soc. Lond. A 371 (1982), 20120213.

LYNDEN-BeLl, D. 1967 Statistical mechanics of violent relaxation in stellar systems. Mon. Not. R. Astron. Soc. 136, 101.

Maffioli, A., Brethouwer, G. \& Lindborg, E. 2016 Mixing efficiency in stratified turbulence. J. Fluid Mech. 794, R3.

Majda, A. \& WAng, X. 2006 Nonlinear Dynamics and Statistical Theories for Basic Geophysical Flows. Cambridge University Press.

MCEwan, A. D. $1983 a$ Internal mixing in stratified fluids. J. Fluid Mech. 128, 59-80.

McEwAn, A. D. $1983 b$ The kinematics of stratified mixing through internal wavebreaking. J. Fluid Mech. 128, 47-57.

MERRYFIELD, W. J. 1998 Effects of stratification on quasi-geostrophic inviscid equilibria. J. Fluid Mech. 354, 345-356.

Michel, J. \& Robert, R. 1994 Large deviations for young measures and statistical mechanics of infinite dimensional dynamical systems with conservation law. Commun. Math. Phys. 159 (1), $195-215$.

Miller, J. 1990 Statistical mechanics of Euler equations in two-dimensions. Phys. Rev. Lett. 65 (17), 2137.

Naso, A., Monchaux, R., Chavanis, P.-H. \& Dubrulle, B. 2010 Statistical mechanics of beltrami flows in axisymmetric geometry: Theory reexamined. Phys. Rev. E 81 (6), 066318.

Obukhov, A. M. 1949 Structure of the temperature field in turbulent flows. Izv. Akad. Nauk SSSR Geogr. Geofiz. 13, 58-69.

Onsager, L. 1949 Statistical hydrodynamics. Il Nuovo Cimento 6, 279-287.

OsBORn, T. R. 1980 Estimates of the local rate of vertical diffusion from dissipation measurements. J. Phys. Oceanogr. 10 (1), 83-89.

Peltier, W. R. \& CAulfield, C. P. 2003 Mixing efficiency in stratified shear flows. Annu. Rev. Fluid Mech. 35 (1), 135-167.

PomeaU, Y. 1994 Statistical approach (to 2D turbulence). In Turbulence: A Tentative Dictionary (ed. O. Cardoso \& P. Tabeling), pp. 385-447. Plenum. 
Pope, S. B. 1985 Pdf methods for turbulent reactive flows. Prog. Energy Combust. Sci. 11 (2), 119-192.

Potters, M., Vaillant, T. \& Bouchet, F. 2013 Sampling microcanonical measures of the 2d Euler equations through Creutz's algorithm: a phase transition from disorder to order when energy is increased. J. Stat. Mech. 2013 (02), P02017.

Prastowo, T., Griffiths, R. W., Hughes, G. \& Hogg, A. M. 2008 Mixing efficiency in controlled exchange flows. J. Fluid Mech. 600, 235-244.

Prieto, R. \& Schubert, W. H. 2001 Analytical predictions for zonally symmetric equilibrium states of the stratospheric polar vortex. J. Atmos. Sci. 58 (18), 2709-2728.

Rehmann, C. R. \& Koseff, J. R. 2004 Mean potential energy change in stratified grid turbulence. Dyn. Atmos. Oceans 37 (4), 271-294.

Renaud, A., Venaille, A. \& Bouchet, F. 2016 Equilibrium statistical mechanics and energy partition for the shallow water model. J. Stat. Phys. 163 (4), 784-843.

Robert, R. \& Sommeria, J. 1991 Statistical equilibrium states for two-dimensional flows. J. Fluid Mech. 229, 291-310.

Robert, R. \& Sommeria, J. 1992 Relaxation towards a statistical equilibrium state in two-dimensional perfect fluid dynamics. Phys. Rev. Lett. 69 (19), 2776.

SAlehipour, H. \& PeltieR, W. R. 2015 Diapycnal diffusivity, turbulent prandtl number and mixing efficiency in boussinesq stratified turbulence. J. Fluid Mech. 775, 464-500.

Salmon, R. 1998 Lectures on Geophysical Fluid Dynamics, vol. 378. Oxford University Press.

SAlmon, R. 2012 Statistical mechanics and ocean circulation. Commun. Nonlinear Sci. Numer. Simul. 17 (5), 2144-2152.

SCHECTER, D. A. 2003 Maximum entropy theory and the rapid relaxation of three-dimensional quasi-geostrophic turbulence. Phys. Rev. E 68 (6), 066309.

Shepherd, T. G. 1993 A unified theory of available potential energy 1. Atmos.-Ocean 31 (1), 1-26.

Shin, L. H., Koseff, J. R., Ivey, G. N. \& Ferziger, J. H. 2005 Parameterization of turbulent fluxes and scales using homogeneous sheared stably stratified turbulence simulations. J. Fluid Mech. 525, 193-214.

Shraiman, B. I. \& Siggia, E. D. 2000 Scalar turbulence. Nature 405 (6787), 639-646.

Sommeria, J. 2001 Two-dimensional turbulence. In New Trends in Turbulence Turbulence: Nouveaux Aspects, pp. 385-447. Springer.

SReenivasan, K. R. 1996 The passive scalar spectrum and the obukhov-corrsin constant. Phys. Fluids 8 (1), 189-196.

Staquet, C. \& Sommeria, J. 2002 Internal gravity waves: from instabilities to turbulence. Annu. Rev. Fluid Mech. 34 (1), 559-593.

Stretch, D. D., Rottman, J. W., Venayagamoorthy, S. K., Nomura, K. K. \& Rehmann, C. R. 2010 Mixing efficiency in decaying stably stratified turbulence. Dyn. Atmos. Oceans 49 (1), 25-36.

Sundaram, R. K. 1996 A First Course in Optimization Theory. Cambridge University Press.

TABAK, E. G. \& TAL, F. A. 2004 Mixing in simple models for turbulent diffusion. Commun. Pure Appl. Maths 57 (5), 563-589.

Tabeling, P. 2002 Two-dimensional turbulence: a physicist approach. Phys. Rep. 362 (1), 1-62.

TAilleuX, R. G. J. 2009 Understanding mixing efficiency in the oceans. Do the nonlinearities of the equation of state for seawater matter? Ocean Sci. 5 (3), 271-283.

Thalabard, S., Dubrulle, B. \& Bouchet, F. 2014 Statistical mechanics of the 3d axisymmetric Euler equations in a Taylor-Couette geometry. J. Stat. Mech. 2014, P01005.

Thalabard, S., Saint-Michel, B., Herbert, É., Daviaud, F. \& Dubrulle, B. 2015 A statistical mechanics framework for the large-scale structure of turbulent von Kármán flows. New J. Phys. 17 (6), 063006.

Thorpe, S. A. 2005 The Turbulent Ocean. Cambridge University Press.

Touchette, H. 2009 The large deviation approach to statistical mechanics. Phys. Rep. 478 (1), $1-69$. 
Townsend, A. A. 1958 The effects of radiative transfer on turbulent flow of a stratified fluid. J. Fluid Mech. 4 (04), 361-375.

Turkington, B., Majda, A., Haven, K.\& DiBattista, M. 2001 Statistical equilibrium predictions of jets and spots on jupiter. Proc. Natl Acad. Sci. USA 98 (22), 12346-12350.

Vallis, G. K. 2006 Atmospheric and Oceanic Fluid Dynamics: Fundamentals and Large-scale Circulation. Cambridge University Press.

Vassilicos, J. C. 2015 Dissipation in turbulent flows. Annu. Rev. Fluid Mech. 47, 95-114.

Venaille, A. 2012 Bottom-trapped currents as statistical equilibrium states above topographic anomalies. J. Fluid Mech. 699, 500-510.

Venaille, A. \& Bouchet, F. 2011 Oceanic rings and jets as statistical equilibrium states. J. Phys. Oceanogr. 41, 1860-1873.

Venaille, A., Dauxois, T. \& Ruffo, S. 2015 Violent relaxation in two-dimensional flows with varying interaction range. Phys. Rev. E 92 (1), 011001.

Venaille, A. \& Sommeria, J. 2010 Modeling mixing in two-dimensional turbulence and stratified fluids. In Proceedings of the IUTAM Symposium on Turbulence in the Atmosphere and Oceans, vol. 28, p. 155. Springer.

Venaille, A., Vallis, G. K. \& Griffies, S. M. 2012 The catalytic role of the beta effect in barotropization processes. J. Fluid Mech. 709, 490-515.

Venayagamoorthy, S. K. \& KosefF, J. R. 2016 On the flux Richardson number in stably stratified turbulence. J. Fluid Mech. 798, R1.

Waite, M. L. \& Bartello, P. 2004 Stratified turbulence dominated by vortical motion. J. Fluid Mech. 517, 281-308.

Warhaft, Z. 2000 Passive scalars in turbulent flows. Annu. Rev. Fluid Mech. 32 (1), 203-240.

WARN, T. 1986 Statistical mechanical equilibria of the shallow water equations. Tellus A 38 (1).

WeICHMAN, P. B. 2006 Equilibrium theory of coherent vortex and zonal jet formation in a system of nonlinear Rossby waves. Phys. Rev. E 73 (3), 036313.

Weichman, P. B. \& Petrich, D. M. 2001 Statistical equilibrium solutions of the shallow water equations. Phys. Rev. Lett. 86, 1761-1764.

Winters, K. B., Lombard, P. N., Riley, J. J. \& D’Asaro, E. A. 1995 Available potential energy and mixing in density-stratified fluids. J. Fluid Mech. 289, 115-128.

Wunsch, S. C. \& FERRARI, R. 2004 Vertical mixing, energy, and the general circulation of the oceans. Annu. Rev. Fluid Mech. 36, 281-314. 